\title{
On Convergence with respect to an Ideal and a Family of Matrices
}

\author{
Jan-David Hardtke \\ Department of Mathematics, Free University of Berlin, Arnimallee 6, 14195 Berlin, Germany \\ Correspondence should be addressed to Jan-David Hardtke; hardtke@math.fu-berlin.de
}

Received 27 November 2013; Accepted 25 March 2014; Published 24 April 2014

Academic Editor: Shamsul Qamar

Copyright (C) 2014 Jan-David Hardtke. This is an open access article distributed under the Creative Commons Attribution License, which permits unrestricted use, distribution, and reproduction in any medium, provided the original work is properly cited.

\begin{abstract}
P. Das et al. recently introduced and studied the notions of strong $A^{I}$-summability with respect to an Orlicz function $F$ and $A^{I}$ statistical convergence, where $A$ is a nonnegative regular matrix and $I$ is an ideal on the set of natural numbers. In this paper, we will generalise these notions by replacing $A$ with a family of matrices and $F$ with a family of Orlicz functions or moduli and study the thus obtained convergence methods. We will also give an application in Banach space theory, presenting a generalisation of Simons' sup-limsup-theorem to the newly introduced convergence methods (for the case that the filter generated by the ideal $I$ has a countable base), continuing some of the author's previous work.
\end{abstract}

\section{Introduction}

Let us begin by recalling that an ideal $I$ on a nonempty set $Y$ is a nonempty set of subsets of $Y$ such that $Y \notin I$ and $I$ is closed under the formation of subsets and finite unions. The ideal is called admissible if $\{y\} \in I$ for each $y \in Y$. For example, if $Y$ is infinite, then the set of all finite subsets of $Y$ forms an ideal on $Y$. If $I$ is an ideal, then $\mathscr{F}(I):=\{Y \backslash A: A \in I\}$ is a filter on $Y$.

Now if $\left(x_{n}\right)_{n \in \mathbb{N}}$ is a sequence in a topological space $X$ and $I$ is an ideal on the set $\mathbb{N}$ of natural numbers, then $\left(x_{n}\right)_{n \in \mathbb{N}}$ is said to be $I$-convergent to $x \in X$ if for every neighbourhood $U$ of $x$ the set $\left\{n \in \mathbb{N}: x_{n} \notin U\right\}$ belongs to $I$ (equivalently, $\{n \in \mathbb{N}$ : $\left.\left.x_{n} \in U\right\} \in \mathscr{F}(I)\right)$. In a Hausdorff space the $I$-limit is unique if it exists. It will be denoted by $I-\lim x_{n}$. If $I_{f}$ is the ideal of all finite subsets of $\mathbb{N}$, then $I_{f}$-convergence is equivalent to the usual convergence. Thus if $I$ is admissible, the usual convergence implies $I$-convergence. For a normed space $X$ the set of all $I$-convergent sequences in $X$ is a subspace of $X^{\mathbb{N}}$ and the map $\left(x_{n}\right) \mapsto I-\lim x_{n}$ is linear. We refer the reader to [1-4] for more information on $I$-convergence.

Recall now that for a given infinite matrix $A=\left(a_{n k}\right)_{n, k \in \mathbb{N}}$ with real or complex entries a sequence $s=\left(s_{k}\right)_{k \in \mathbb{N}}$ of (real or complex) numbers is said to be $A$-summable to the number $a$ provided that each of the series $\sum_{k=1}^{\infty} a_{n k} s_{k}$ is convergent and $\lim _{n \rightarrow \infty} \sum_{k=1}^{\infty} a_{n k} s_{k}=a$.

The matrix $A$ is called regular if every sequence that is convergent in the ordinary sense is also $A$-summable to the same limit. A well-known theorem of Toeplitz states that $A$ is regular if and only if the following holds:

(i) $\sup _{n \in \mathbb{N}} \sum_{k=1}^{\infty}\left|a_{n k}\right|<\infty$,

(ii) $\lim _{n \rightarrow \infty} \sum_{k=1}^{\infty} a_{n k}=1$,

(iii) $\lim _{n \rightarrow \infty} a_{n k}=0 \forall k \in \mathbb{N}$.

Let us suppose for the moment that $A$ is regular and also nonnegative (i.e., $a_{n k} \geq 0$ for all $n, k \in \mathbb{N}$ ). We will denote by $D(s, a, \varepsilon)$ the set $\left\{k \in \mathbb{N}:\left|s_{k}-a\right| \geq \varepsilon\right\}$ for every $\varepsilon>0$. Then $s$ is said to be $A$-statistically convergent to $a$ if for every $\varepsilon>0$ we have $\lim _{n \rightarrow \infty} \sum_{k=1}^{\infty} a_{n k} \chi_{D(s, a, \varepsilon)}(k)=0$, where the symbol $\chi_{K}$ denotes the characteristic function of the set $K \subseteq \mathbb{N}$. If one takes $A$ to be the Cesàro matrix (i.e., $a_{n k}=1 / n$ for $k \leq n$ and $a_{n k}=0$ for $k>n$ ) one gets the usual notion of statistical convergence as it was introduced by Fast in [5]. Note that the set $I_{A}$ of all subsets $K \subseteq \mathbb{N}$ for which $\lim _{n \rightarrow \infty} \sum_{k=1}^{\infty} a_{n k} \chi_{K}(k)=0$ holds is an ideal on $\mathbb{N}$ and $A$-statistical convergence is nothing but convergence with respect to this ideal. 
For any number $p>0$ the sequence $s$ is said to be strongly $A$ - $p$-summable to $a$ provided that $\sum_{k=1}^{\infty} a_{n k}\left|s_{k}-a\right|^{p}<\infty$ for all $n \in \mathbb{N}$ and $\lim _{n \rightarrow \infty} \sum_{k=1}^{\infty} a_{n k}\left|s_{k}-a\right|^{p}=0$. The strong $A$ - $p$-summability is a linear consistent summability method and the strong $A$ - $p$-limit is uniquely determined whenever it exists. In [6] Connor proved that $s$ is statistically convergent to $a$ whenever it is strongly $p$-Cesàro convergent to $a$ and the converse is true if $s$ is bounded. Practically the same proof given in [6] still works if one replaces the Cesàro matrix by an arbitrary nonnegative regular matrix $A$. In particular, strong $A$ - $p$-summability and $A$-statistical convergence are equivalent on bounded sequences (see also [7, Theorem $8]$ ). More information on strong matrix summability can be found in [8] (for the case $p=1$ ) or [9].

In [10] Maddox proposed a generalisation of strong $A$ $p$-summability by replacing the number $p$ with a sequence $\mathbf{p}=\left(p_{k}\right)_{k \in \mathbb{N}}$ of positive numbers: the sequence $s$ is strongly $A$-p-summable to $a$ if $\sum_{k=1}^{\infty} a_{n k}\left|s_{k}-a\right|^{p_{k}}<\infty$ for every $n \in \mathbb{N}$ and $\lim _{n \rightarrow \infty} \sum_{k=1}^{\infty} a_{n k}\left|s_{k}-a\right|^{p_{k}}=0$.

Next, let us recall that a function $F:[0, \infty) \rightarrow[0, \infty)$ is called an Orlicz function if it is increasing, continuous, and convex and satisfies $\lim _{t \rightarrow \infty} F(t)=\infty$ as well as $F(t)=0$ if and only if $t=0$. If we drop the convexity and replace it by the condition $F(s+t) \leq F(s)+F(t)$ for all $s, t \geq 0$, then $F$ is called a modulus. For example, the function $F_{p}$ defined by $F_{p}(t)=t^{p}$ is an Orlicz function for $p \geq 1$ and a modulus for $0<p \leq 1$. We will denote the set of all Orlicz functions by $\mathcal{O}$ and the set of all moduli by $\mathscr{M}$.

Connor introduced another generalisation of strong matrix summability in [7]: if $F$ is a modulus, then $s$ is said to be strongly $A$-summable to the limit $a$ with respect to $F$ if $\sum_{k=1}^{\infty} a_{n k} F\left(\left|s_{k}-a\right|\right)<\infty$ for all $n \in \mathbb{N}$ and $\lim _{n \rightarrow \infty} \sum_{k=1}^{\infty} a_{n k} F\left(\left|s_{k}-a\right|\right)=0$. It is shown in [7, Theorem $8]$ that strong $A$-summability with respect to $F$ implies $A$-statistical convergence and that the converse holds for bounded sequences. In [11] Demirci replaced the modulus in Connor's definition by an Orlicz function and studied which results carry over to this setting.

Another common generalised convergence method is that of almost convergence introduced by Lorentz in [12]. For this we first recall that a Banach limit is a linear functional $L$ on the space $\ell^{\infty}$ of all bounded real-valued sequences such that $L$ is shift-invariant (i.e., $\left.L\left(\left(s_{n+1}\right)_{n \in \mathbb{N}}\right)=L\left(\left(s_{n}\right)_{n \in \mathbb{N}}\right)\right)$, positive (i.e., $L\left(\left(s_{n}\right)_{n \in \mathbb{N}}\right) \geq 0$ if $s_{n} \geq 0$ for all $n$ ), and fulfills $L(1,1, \ldots)=1$. The existence of a Banach limit can be easily proved by means of the Hahn-Banach extension theorem. A sequence $s \in \ell^{\infty}$ is said to be almost convergent to $a \in \mathbb{R}$ if $L(s)=a$ for every Banach limit $L$.

It is proved in [12] that almost convergence is equivalent to "uniform Cesàro convergence." More precisely, a bounded sequence $s=\left(s_{k}\right)_{k \in \mathbb{N}}$ in $\mathbb{R}$ is almost convergent to $a \in \mathbb{R}$ if and only if the following holds:

$$
\frac{1}{n} \sum_{k=1}^{n} s_{k+i} \stackrel{n \rightarrow \infty}{\longrightarrow} a \quad \text { uniformly in } i \in \mathbb{N}_{0},
$$

where $\mathbb{N}_{0}=\mathbb{N} \cup\{0\}$.

Lorentz subsequently introduced and studied the notion of $F_{A}$-convergence by replacing the Cesàro matrix with an arbitrary real-valued regular matrix $A$ : a bounded sequence $s=\left(s_{k}\right)_{k \in \mathbb{N}}$ in $\mathbb{R}$ is said to be $F_{A}$-convergent to $a \in \mathbb{R}$ provided that

$$
\sum_{k=1}^{\infty} a_{n k} s_{k+i} \stackrel{n \rightarrow \infty}{\longrightarrow} a \quad \text { uniformly in } i \in \mathbb{N}_{0}
$$

Stieglitz further generalised the notion of almost convergence in the following way (cf. [13]): consider a sequence $\mathscr{B}=$ $\left(B_{i}\right)_{i \in \mathbb{N}_{0}}=\left(\left(b_{n k}^{(i)}\right)_{n, k \in \mathbb{N}}\right)_{i \in \mathbb{N}_{0}}$ of matrices with entries in $\mathbb{R}$ or $\mathbb{C}$ and a bounded sequence $s=\left(s_{k}\right)_{k \in \mathbb{N}}$ of real or complex numbers. Then $s$ is said to be $F_{\mathscr{B}}$-convergent to the number $a$ if each of the series $\sum_{k=1}^{\infty} b_{n k}^{(i)} s_{k}$ with $n \in \mathbb{N}, i \in \mathbb{N}_{0}$ is convergent and

$$
\sum_{k=1}^{\infty} b_{n k}^{(i)} s_{k} \stackrel{n \rightarrow \infty}{\longrightarrow} a \quad \text { uniformly in } i \in \mathbb{N}_{0} .
$$

To obtain $F_{A}$-convergence, take $b_{n k}^{(i)}=a_{n k-i}$ for $k>i$ and $b_{n k}^{(i)}=0$ for $k \leq i$.

Maddox introduced the $F_{\mathscr{B}}$-analogue of strong matrix summability in [14]. If each of the matrices $B_{i}$ is nonnegative and $s=\left(s_{k}\right)_{k \in \mathbb{N}}$ is a (not necessarily bounded) sequence in $\mathbb{R}$ or $\mathbb{C}$, then $s$ is said to be strongly $F_{\mathscr{B}}$-convergent to $a$ provided that

$$
\sum_{k=1}^{\infty} b_{n k}^{(i)}\left|s_{k}-a\right| \stackrel{n \rightarrow \infty}{\longrightarrow} 0 \quad \text { uniformly in } i \in \mathbb{N}_{0}
$$

Very recently, the authors of [15] introduced the following definitions, combining matrices and ideals.

Definition 1 (cf. [15]). Let $A=\left(a_{n k}\right)_{n, k \in \mathbb{N}}$ be a nonnegative regular matrix, $I$ an ideal on $\mathbb{N}$, and $F$ an Orlicz function. Let $a$ be any real or complex number. A sequence $s=\left(s_{k}\right)_{k \in \mathbb{N}}$ in $\mathbb{R}$ or $\mathbb{C}$ is said to be

(i) strongly $A^{I}$-summable to $a$ with respect to $F$ if

$$
I-\lim \sum_{k=1}^{\infty} a_{n k} F\left(\left|s_{k}-a\right|\right)=0,
$$

(ii) $A^{I}$-statistically convergent to $a$ if

$$
I-\lim \sum_{k=1}^{\infty} a_{n k} \chi_{D(s, a, \varepsilon)}(k)=0
$$

for every $\varepsilon>0$.

It is proved in [15, Theorem 2.5$]$ that $A^{I}$-summability with respect to $F$ implies $A^{I}$-statistical convergence (to the same limit) and the converse holds if the sequence $s$ is bounded and $F$ satisfies the $\Delta_{2}$-condition (i.e., there is a constant $K$ such that $F(2 t) \leq K F(t)$ for all $t \geq 0)$.

We would like to propose here the following three definitions that include all the above mentioned generalised convergence methods. 
First we define a sequence $\left(g_{n}\right)_{n \in \mathbb{N}}$ of functions from a set $S$ into a generalised metric space $(X, d)$ (same as a metric space except that $d$ is allowed to take values in $[0, \infty]$; for example, $d(a, b)=|a-b|$ for $a, b \in[0, \infty), d(a, \infty)=$ $d(\infty, a)=\infty$ for all $a \in[0, \infty)$, and $d(\infty, \infty)=0$ defines a generalised metric on $[0, \infty]$ ) to be uniformly convergent to the function $g: S \rightarrow X$ along the ideal $I$ if for every $\varepsilon>0$ there is some $E \in I$ such that for every $s \in S$

$$
\left\{n \in \mathbb{N}: d\left(g_{n}(s), g(s)\right) \geq \varepsilon\right\} \subseteq E
$$

or, equivalently, for every $\varepsilon>0$, we have

$$
\left\{n \in \mathbb{N}: \sup _{s \in S} d\left(g_{n}(s), g(s)\right) \geq \varepsilon\right\} \in I .
$$

If $I=I_{f}$, this yields the usual definition of uniform convergence. Also, this definition is a direct generalisation of the definition of $\mu$-statistical uniform convergence given in [16]. The uniform convergence of $\left(g_{n}\right)_{n \in \mathbb{N}}$ to $g$ along I clearly implies $I-\lim g_{n}(s)=g(s)$ for all $s \in S$.

Now we come to the main definition.

Definition 2. Let $I$ be an ideal on $\mathbb{N}$ and $S$ any nonempty set. Let $\mathscr{B}=\left(B_{i}\right)_{i \in S}=\left(\left(b_{n k}^{(i)}\right)_{n, k \in \mathbb{N}}\right)_{i \in S}$ be a family of (not necessarily regular) matrices with entries in $\mathbb{R}$ or $\mathbb{C}$ and $\mathscr{F}=$ $\left(F_{k}^{(i)}\right)_{k \in \mathbb{N}, i \in S}$ a family in $\mathscr{M} \cup \mathcal{O}$. Suppose that there is some $i_{0} \in S$ such that

$$
\inf _{n \in \mathbb{N}} \sum_{k=1}^{\infty}\left|b_{n k}^{\left(i_{0}\right)}\right|>0
$$

Finally, let $s=\left(s_{k}\right)_{k \in \mathbb{N}}$ be a sequence in $\mathbb{R}$ or $\mathbb{C}$ and $a \in \mathbb{R}$ or $\mathbb{C}$.

(i) $s$ is said to be $\mathscr{B}^{I}$-summable to $a$ provided that each of the series $\sum_{k=1}^{\infty} b_{n k}^{(i)} s_{k}$ is convergent and

$$
I-\lim \sum_{k=1}^{\infty} b_{n k}^{(i)} s_{k}=a \quad \text { uniformly in } i \in S
$$

(ii) If each matrix $B_{i}$ is nonnegative, then $s$ is said to be strongly $\mathscr{B}^{I}$-summable to $a$ with respect to $\mathscr{F}$ if

$$
I-\lim \sum_{k=1}^{\infty} b_{n k}^{(i)} F_{k}^{(i)}\left(\left|s_{k}-a\right|\right)=0 \quad \text { uniformly in } i \in S .
$$

(iii) If each $B_{i}$ is nonnegative, then $s$ is said to be $\mathscr{B}^{I}$ statistically convergent to $a$ provided that for every $\varepsilon>0$

$$
I-\lim \sum_{k=1}^{\infty} b_{n k}^{(i)} \chi_{D(s, a, \varepsilon)}(k)=0 \quad \text { uniformly in } i \in S .
$$

If $F_{k}^{(i)}=\mathrm{id}_{[0, \infty)}$ for all $k \in \mathbb{N}, i \in S$ in (ii) we simply speak of strong $\mathscr{B}^{I}$-summability. Clearly, strong $\mathscr{B}^{I}$-summability to a implies $\mathscr{B}^{I}$-summability to $a$ provided that $s$ is bounded, $\sum_{k=1}^{\infty} b_{n k}^{(i)}<\infty$ for all $k \in \mathbb{N}, i \in S$ and

$$
I-\lim \sum_{k=1}^{\infty} b_{n k}^{(i)}=1 \quad \text { uniformly in } i \in S .
$$

Taking $B_{i}=A$ and $F_{k}^{(i)}=F \in \mathcal{O}$ for each $i \in S$ and $k \in \mathbb{N}$ in (ii) and (iii) yields the definitions of strong $A^{I}$-summability with respect to $F$ and of $A^{I}$-statistical convergence. If we take $I=I_{f}$ and $S=\mathbb{N}_{0}$ in (i) and (ii) we obtain the definitions of $F_{\mathscr{B}}$ - and strong $F_{\mathscr{B}}$-convergence. Setting $I=I_{f}, B_{i}=A$ for every $i \in S$ and $F_{k}^{(i)}=F_{p_{k}}$ for all $i \in S, k \in \mathbb{N}$ in (ii) gives us the definition of Maddox's strong $A$-p-summability.

Note also that if each $B_{i}$ is nonnegative, then the set $J_{\mathscr{B}, I}$ of all subsets $K \subseteq \mathbb{N}$, such that

$$
I-\lim \sum_{k=1}^{\infty} b_{n k}^{(i)} \chi_{K}(k)=0 \quad \text { uniformly in } i \in S,
$$

is an ideal on $\mathbb{N}$ (the condition (+) ensures $\mathbb{N} \notin J_{\mathscr{B}, I}$ ). The $\mathscr{B}^{I}$ statistical convergence is nothing but the convergence with respect to $J_{\mathscr{B}, I}$. In the case that $B_{i}$ is the infinite unit matrix for each $i \in S$ we have $J_{\mathscr{B}, I}=I$.

In the next section we will start to investigate the above convergence methods.

\section{Some Convergence Theorems}

If not otherwise stated, we will denote by $I$ an ideal on $\mathbb{N}$, by $\mathscr{B}=\left(B_{i}\right)_{i \in S}=\left(\left(b_{n k}^{(i)}\right)_{n, k \in \mathbb{N}}\right)_{i \in S}$ a family of real or complex matrices (where $S$ is any nonempty index set) such that there is some $i_{0} \in S$ with $(+)$, and by $\mathscr{F}=\left(F_{k}^{(i)}\right)_{k \in \mathbb{N}, i \in S}$ a family in $\mathscr{M} \cup \mathcal{O}$. Finally, $s=\left(s_{k}\right)_{k \in \mathbb{N}}$ denotes a sequence in $\mathbb{R}$ or $\mathbb{C}$ and $a$ an element of $\mathbb{R}$ or $\mathbb{C}$, as in the previous section.

The following two propositions (wherein each $B_{i}$ is implicitly assumed to be nonnegative) generalise the aforementioned results from [15, Theorem 2.5]. The techniques used there followed the line of [17] while we will adopt the techniques from [6].

Proposition 3. Suppose that $s$ is strongly $\mathscr{B}^{I}$-summable to a with respect to $\mathscr{F}$ and that

$$
L(t):=\inf \left\{F_{k}^{(i)}(t): k \in \mathbb{N}, i \in S\right\}>0 \quad \forall t>0 .
$$

Then $s$ is also $\mathscr{B}^{I}$-statistically convergent to a.

Proof. Let $\varepsilon, \delta>0$ be arbitrary. By assumption there is some $E \in I$ such that for all $i \in S$

$$
\left\{n \in \mathbb{N}: \sum_{k=1}^{\infty} b_{n k}^{(i)} F_{k}^{(i)}\left(\left|s_{k}-a\right|\right) \geq \delta L(\varepsilon)\right\} \subseteq E .
$$

But we have

$$
\begin{aligned}
\sum_{k=1}^{\infty} b_{n k}^{(i)} F_{k}^{(i)}\left(\left|s_{k}-a\right|\right) & \geq \sum_{k=1}^{\infty} b_{n \mathrm{k}}^{(i)} F_{k}^{(i)}\left(\left|s_{k}-a\right|\right) \chi_{D(s, a, \varepsilon)}(k) \\
& \geq L(\varepsilon) \sum_{k=1}^{\infty} b_{n k}^{(i)} \chi_{D(s, a, \varepsilon)}(k)
\end{aligned}
$$


for all $i \in S, k \in \mathbb{N}$. Hence

$$
\left\{n \in \mathbb{N}: \sum_{k=1}^{\infty} b_{n k}^{(i)} \chi_{D(s, a, \varepsilon)}(k) \geq \delta\right\} \subseteq E
$$

for every $i \in S$ and the proof is finished.

Proposition 4. Suppose that sis bounded and $\mathscr{B}^{I}$-statistically convergent to a. If $\mathscr{F}$ is equicontinuous at 0 and there exists an $A \in I$ such that

$$
M:=\sup \left\{\sum_{k=1}^{\infty} b_{n k}^{(i)}: n \in \mathbb{N} \backslash A, i \in S\right\}<\infty,
$$

as well as

$$
h(t):=\sup \left\{F_{k}^{(i)}(t): k \in \mathbb{N}, i \in S\right\}<\infty \quad \forall t \geq 0,
$$

then $s$ is also strongly $B^{I}$-summable to a with respect to $\mathscr{F}$.

Proof. Let $\varepsilon>0$ be arbitrary. Take $\tau>0$ with $\tau\left(M+h\left(\|s\|_{\infty}+\right.\right.$ $|a|))<\varepsilon$. Since $\mathscr{F}$ is equicontinuous at 0 , we can find a $\delta>0$ such that $F_{k}^{(i)}(t) \leq \tau$ for all $t \in[0, \delta]$ and all $k \in \mathbb{N}, i \in S$.

Because $s$ is $\mathscr{B}^{I}$-statistically convergent to $a$ there is some $E \in I$ such that for every $i \in S$

$$
\left\{n \in \mathbb{N}: \sum_{k=1}^{\infty} b_{n k}^{(i)} \chi_{D(s, a, \delta)}(k) \geq \tau\right\} \subseteq E .
$$

It follows that for every $n \in \mathbb{N} \backslash(E \cup A)$ and all $i \in S$

$$
\begin{aligned}
\sum_{k=1}^{\infty} b_{n k}^{(i)} F_{k}^{(i)}\left(\left|s_{k}-a\right|\right) & \\
\leq & \tau \sum_{k=1}^{\infty} b_{n k}^{(i)} \chi_{\mathbb{N} \backslash D(s, a, \delta)}(k) \\
& +\sum_{k=1}^{\infty} b_{n k}^{(i)} F_{k}^{(i)}\left(\left|s_{k}-a\right|\right) \chi_{D(s, a, \delta)}(k) \\
\leq & \tau M+h\left(\|s\|_{\infty}+|a|\right) \sum_{k=1}^{\infty} b_{n k}^{(i)} \chi_{D(s, a, \delta)}(k) \\
\leq & \tau\left(M+h\left(\|s\|_{\infty}+|a|\right)\right)<\varepsilon
\end{aligned}
$$

and we are done.

So in particular, if $\mathscr{B}$ and $\mathscr{F}$ meet the requirements of both Propositions 3 and 4 , then $\mathscr{B}^{I}$-statistical convergence and strong $\mathscr{B}^{I}$-summability with respect to $\mathscr{F}$ coincide on bounded sequences. Note that all the assumptions on $\mathscr{F}$ are satisfied if $F_{k}^{(i)}=F_{p_{k i}}$ for a family $\left(p_{k i}\right)_{k \in \mathbb{N}, i \in S}$ of positive numbers which is bounded and bounded away from zero.

If $I \subseteq J_{\mathscr{B}, I}$, in other words, if

$$
I-\lim \sum_{k=1}^{\infty} b_{n k}^{(i)} \chi_{A}(k)=0 \quad \text { uniformly in } i \in S \forall A \in I \text {, }
$$

then $I$-convergence implies $\mathscr{B}^{I}$-statistical convergence (to the same limit). Thus if $\mathscr{B}$ and $\mathscr{F}$ additionally satisfy the requirements of Proposition 4 , then for bounded sequences $I$-convergence also implies strong $\mathscr{B}^{I}$-summability to the same limit. Concerning the consistency of ordinary $\mathscr{B}^{I}$ summability, we have the following sufficient conditions which are analogous to those of Toeplitz's theorem. We write $d_{I}$ for the set of all bounded sequences $\left(a_{k}\right)_{k \in \mathbb{N}}$ for which $\left\{k \in \mathbb{N}: a_{k} \neq 0\right\} \in I$.

Lemma 5. Suppose that $\sum_{k=1}^{\infty}\left|b_{n k}^{(i)}\right|<\infty$ for all $n \in \mathbb{N}, i \in S$ and

$$
\exists A \in I \quad M:=\sup \left\{\sum_{k=1}^{\infty}\left|b_{n k}^{(i)}\right|: n \in \mathbb{N} \backslash A, i \in S\right\}<\infty
$$

$I-\lim \sum_{k=1}^{\infty} b_{n k}^{(i)} a_{k}=0 \quad$ uniformly in $i \in S \forall\left(a_{k}\right) \in d_{I}$,

$$
I-\lim \sum_{k=1}^{\infty} b_{n k}^{(i)}=1 \quad \text { uniformlyin } i \in S
$$

Then for every bounded sequence $s=\left(s_{n}\right)_{n \in \mathbb{N}}$ in $\mathbb{R}$ or $\mathbb{C}$, if $I$ - $\lim s_{n}=a$, then $s$ is also $\mathscr{B}^{I}$-summable to $a$.

Proof. Because of (25) we may assume $a=0$. Let $\varepsilon>0$ be arbitrary. Since $I$-lims $s_{n}=0$, we have $C:=\left\{n \in \mathbb{N}:\left|s_{n}\right| \geq \varepsilon\right\} \in$ $I$ and hence by (24) there is some $E \in I$ such that

$$
\left\{n \in \mathbb{N}:\left|\sum_{k=1}^{\infty} b_{n k}^{(i)} s_{k} \chi_{C}(k)\right| \geq \varepsilon\right\} \subseteq E \quad \forall i \in S .
$$

But for all $i \in S$ and all $n \in \mathbb{N} \backslash A$

$$
\begin{aligned}
\left|\sum_{k=1}^{\infty} b_{n k}^{(i)} s_{k}\right| \leq & \left|\sum_{k=1}^{\infty} b_{n k}^{(i)} s_{k} \chi_{C}(k)\right| \\
& +\sum_{k=1}^{\infty}\left|b_{n k}^{(i)}\right| \chi_{\mathbb{N} \mid C}(k)\left|s_{k}\right| \\
\leq & \left|\sum_{k=1}^{\infty} b_{n k}^{(i)} s_{k} \chi_{C}(k)\right|+M \varepsilon,
\end{aligned}
$$

and thus

$$
\left\{n \in \mathbb{N}:\left|\sum_{k=1}^{\infty} b_{n k}^{(i)} s_{k}\right| \geq \varepsilon(1+M)\right\} \subseteq E \cup A \quad \forall i \in S
$$

and we are done.

The next proposition is the direct generalisation of [18, Theorem 3.3] to our setting. Its proof is easy and moreover virtually the same as in [18] so it will be omitted. 
Proposition 6. Suppose that we are given two families of nonnegative matrices $\mathscr{B}=\left(\left(b_{n k}^{(i)}\right)_{n, k \in \mathbb{N}}\right)_{i \in S}$ and $\mathscr{A}=\left(\left(a_{n k}^{(i)}\right)_{n, k \in \mathbb{N}}\right)_{i \in S}$. If

$$
I-\lim \sum_{k=1}^{\infty}\left|a_{n k}^{(i)}-b_{n k}^{(i)}\right|=0 \quad \text { uniformly in } i \in S,
$$

then $J_{\mathscr{B}, I}=J_{\mathscr{A}, I}$.

In [19] it was proved that a bounded (real) sequence $s$ is statistically convergent to $a$ if and only if $s$ is Cesàro-summable to $a$ and the "variance" $\sigma_{n}(s)^{2}$ := $1 / n \sum_{i=1}^{n}\left(a-1 / n \sum_{k=1}^{n} s_{k}\right)^{2}$ converges to 0 . The proposition below is a generalisation of this result. We will use the notation

$$
\sigma_{n i}^{\mathscr{B}, \mathscr{F}}(s):=\sum_{k=1}^{\infty} b_{n k}^{(i)} F_{k i}\left(\left|s_{k}-\left(B_{i} s\right)(n)\right|\right),
$$

provided that each $B_{i}$ is nonnegative.

First we need the following lemma, whose proof is analogous to those of Propositions 3 and 4 and will therefore be omitted.

Lemma 7. Suppose that $\mathscr{F}$ and $\mathscr{B}$ fulfill the requirements of Propositions 3 and 4 and let $y=\left(y_{n i}\right)_{n \in \mathbb{N}, i \in S}$ be a family in $\mathbb{R}$ or $\mathbb{C}$. Put $A_{\varepsilon, n, i}:=D\left(s, y_{n i}, \varepsilon\right)$ for all $i \in S, n \in \mathbb{N}$ and $\varepsilon>0$. Then

$$
I-\lim \sum_{k=1}^{\infty} b_{n k}^{(i)} F_{k i}\left(\left|s_{k}-y_{n i}\right|\right)=0 \quad \text { uniformly in } i \in S
$$

implies that for every $\varepsilon>0$

$$
I-\lim \sum_{k=1}^{\infty} b_{n k}^{(i)} \chi_{A_{\varepsilon, n, i}}(k)=0 \quad \text { uniformly in } i \in S
$$

and the converse is true if $s$ is bounded and $\sup _{i \in \mathbb{N}, n \in \mathbb{N} \mid V}\left|y_{n i}\right|<$ $\infty$ for some $V \in I$.

Proposition 8. Let $s$ be bounded. Under the same hypotheses as in the previous lemma and the additional assumption that $\sum_{k=1}^{\infty}\left|b_{n k}^{(i)}\right|<\infty$ for all $n \in \mathbb{N}, i \in S$ and

$$
I-\lim \sum_{k=1}^{\infty} b_{n k}^{(i)}=1 \quad \text { uniformlyini } \in S,
$$

$s$ is $\mathscr{B}^{I}$-statistically convergent to the number $a$ if and only if $s$ is $\mathscr{B}^{I}$-summable to $a$ and $\sigma_{n i}^{\mathscr{B}, \mathscr{F}}(s)$ converges to 0 along $I$ uniformly in $i \in S$.

Proof. In view of Lemma 7 it is enough to consider the case $F_{k i}=\mathrm{id}_{[0, \infty)}$ for all $k \in \mathbb{N}, i \in S$. We first assume that $s$ is $\mathscr{B}^{I}$-summable to $a$ and that

$$
\begin{aligned}
& I-\lim \sigma_{n i}^{\mathscr{R}, \mathscr{F}}(s) \\
& =I-\lim \sum_{k=1}^{\infty} b_{n k}^{(i)}\left|s_{k}-\left(B_{i} s\right)(n)\right|=0 \quad \text { uniformly in } i \in S .
\end{aligned}
$$

Because of

$$
\begin{aligned}
\sum_{k=1}^{\infty} b_{n k}^{(i)} \mid & s_{k}-a \mid \\
\leq & \sum_{k=1}^{\infty} b_{n k}^{(i)}\left|s_{k}-\left(B_{i} s\right)(n)\right| \\
& +\sum_{k=1}^{\infty} b_{n k}^{(i)}\left|\left(B_{i} s\right)(n)-a\right| \\
\leq & \sigma_{n i}^{\mathscr{B}, \mathscr{F}}(s)+\left|\left(B_{i} s\right)(n)-a\right| M \quad \forall n \in \mathbb{N} \backslash A, \forall i \in S,
\end{aligned}
$$

where $A$ and $M$ are as in Proposition 4, it follows that $s$ is strongly $\mathscr{B}^{I}$-summable to $a$ and hence by Proposition 3 it is also $\mathscr{B}^{I}$-statistically convergent to $a$.

Conversely, let $s$ be $\mathscr{B}^{I}$-statistically convergent to $a$. Then by Proposition $4 s$ is also strongly $\mathscr{B}^{I}$-summable to $a$ and because of assumption (33) it follows that $s$ is $\mathscr{B}^{I}$-summable to $a$. Moreover, we have

$$
\begin{aligned}
\sigma_{n i}^{\mathscr{B}, \mathscr{F}}(s) & =\sum_{k=1}^{\infty} b_{n k}^{(i)}\left|s_{k}-\left(B_{i} s\right)(n)\right| \\
& \leq \sum_{k=1}^{\infty} b_{n k}^{(i)}\left|s_{k}-a\right|+\sum_{k=1}^{\infty} b_{n k}^{(i)}\left|a-\left(B_{i} s\right)(n)\right| \\
& \leq \sum_{k=1}^{\infty} b_{n k}^{(i)}\left|s_{k}-a\right|+M\left|a-\left(B_{i} s\right)(n)\right|
\end{aligned}
$$

$$
\forall n \in \mathbb{N} \backslash A, \quad \forall i \in S
$$

and hence $\sigma_{n i}^{\mathscr{R}, \mathscr{F}}(s)$ converges to 0 along $I$ uniformly in $i \epsilon$ $S$.

According to [12, Theorem 2], for any regular matrix $A$ the $F_{A}$-convergence of a sequence implies its almost convergence to the same limit and by [12, Theorem 3] the converse is true if $A$ satisfies $\lim _{n \rightarrow \infty} \sum_{k=1}^{\infty}\left|a_{n k}-a_{n k+1}\right|=0$. The following two results are generalisations of these facts. Their proofs remain virtually the same and will not be given here.

Proposition 9. Let $A=\left(a_{n k}\right)_{n, k \in \mathbb{N}}$ be an infinite matrix in $\mathbb{R}$ such that $\sum_{k=1}^{\infty}\left|a_{n k}\right|<\infty$ for all $n \in \mathbb{N}$ and I-lim $\sum_{k=1}^{\infty} a_{n k}=1$. Put $\mathscr{A}=\left(\left(a_{n k}^{(i)}\right)_{n, k \in \mathbb{N}}\right)_{i \in \mathbb{N}_{0}}$, where $a_{n k}^{(i)}=a_{n k-i}$ for $k>i$ and $a_{n k}^{(i)}=0$ for $k \leq i$.

Let $s \in \ell^{\infty}$ be $\mathscr{A}^{I}$-summable to the value $a$. Then $s$ is also almost convergent to $a$.

Theorem 10. Let $A$ and $\mathscr{A}$ be as in the previous proposition but assume additionally that $I-\lim a_{n k}=0$ for every $k \in \mathbb{N}$, $\sup _{n \in \mathbb{N} \mid V} \sum_{k=1}^{\infty}\left|a_{n k}\right|<\infty$ for some $V \in I$, and

$$
I-\lim \sum_{k=1}^{\infty}\left|a_{n k}-a_{n k+1}\right|=0 .
$$


Let $C$ be the Cesàro matrix and suppose that the family $\mathscr{C}$ arises from $C$ as $\mathscr{A}$ from $A$. Suppose further that the ideal I is admissible and that $J$ is another ideal. Let $s \in \ell^{\infty}$ be $\mathscr{C}^{J}$ summable to the value $a$. Then $s$ is also $\mathscr{A}^{I}$-summable to $a$.

In [4] the notion of I-Cauchy sequences in arbitrary metric spaces, which generalises the notion of statistically Cauchy sequences of Fridy (cf. [20]), was introduced. A sequence $\left(x_{n}\right)_{n \in \mathbb{N}}$ in a metric space $(X, d)$ is said to be an $I$ Cauchy sequence if for every $\varepsilon>0$ there is some $k \in \mathbb{N}$ such that $\left\{n \in \mathbb{N}: d\left(x_{n}, x_{k}\right) \geq \varepsilon\right\} \in I$. For $I=I_{f}$ this yields just an equivalent formulation of the notion of an ordinary Cauchy sequence. Fridy's notion of statistically Cauchy sequences is obtained by taking $I=J_{C, I_{f}}$, where $C$ is the Cesàro matrix. It was proved in [4] that every $I$-convergent sequence is $I$ Cauchy (cf. [4, Proposition 1]) and that, in the case of an admissible ideal $I$, the metric space $(X, d)$ is complete if and only if every $I$-Cauchy sequence in $(X, d)$ is $I$-convergent (cf. [4, Theorem 2]). The proof of [4, Theorem 2] also shows that every $I$-convergent sequence possesses a subsequence which is convergent in the ordinary sense.

In [20] it was proved that a sequence of numbers is statistically convergent if and only if it is statistically Cauchy, but a third equivalent condition was obtained there as well; namely, a number sequence $\left(s_{n}\right)_{n \in \mathbb{N}}$ is statistically convergent if and only if there is a sequence $\left(t_{n}\right)_{n \in \mathbb{N}}$ which is convergent in the usual sense and coincides "almost everywhere" with $\left(s_{n}\right)_{n \in \mathbb{N}}$, which in our notation means precisely $\{n \in \mathbb{N}$ : $\left.s_{n} \neq t_{n}\right\} \in J_{C, I_{f}}$.

It is clear that, for any two sequences $\left(x_{n}\right)_{n \in \mathbb{N}},\left(y_{n}\right)_{n \in \mathbb{N}}$ in an arbitrary topological space, if $\left(y_{n}\right)_{n \in \mathbb{N}}$ is $I$-convergent and $\left\{n \in \mathbb{N}: x_{n} \neq y_{n}\right\} \in I$, then $\left(x_{n}\right)_{n \in \mathbb{N}}$ is also $I$-convergent. For the case of $\mathscr{B}^{I}$-statistical convergence of sequences of numbers we can prove a converse result provided that $\mathscr{F}(I)$ has a countable base that fulfills a certain condition with respect to the matrix-family $\mathscr{B}$. The proof uses the basic ideas from [20].

Theorem 11. Let I be an admissible ideal with $I \subseteq J_{\mathscr{B}, I}$ such that there is an increasing sequence $\left(B_{m}\right)_{m \in \mathbb{N}}$ in I for which $\{\mathbb{N} \backslash$ $\left.B_{m}: m \in \mathbb{N}\right\}$ forms a base of $\mathscr{F}(I)$ and

$$
\sup _{i \in S} \sup _{n \in B_{m}} \sum_{k=1}^{\infty} b_{n k}^{(i)} \chi_{\mathbb{N} \backslash B_{m}}(k) \stackrel{m \rightarrow \infty}{\longrightarrow} 0 .
$$

Then the sequence $s=\left(s_{n}\right)_{n \in \mathbb{N}}$ is $\mathscr{B}^{I}$-statistically convergent to $a$ if and only if there is a sequence $\left(t_{n}\right)_{n \in \mathbb{N}}$ which is I-convergent to $a$ and fulfills $\left\{n \in \mathbb{N}: s_{n} \neq t_{n}\right\} \in J_{\mathscr{B}, I}$.

Proof. We only have to show the necessity. So let $s$ be $\mathscr{B}^{I}$ statistically convergent to $a$. Put $\varepsilon_{m}=2^{-m}$ and $A_{m}=\{k \in \mathbb{N}$ : $\left.\left|s_{k}-a\right| \geq \varepsilon_{m}\right\}$ for every $m \in \mathbb{N}$. Then for every $m \in \mathbb{N}$ there exists a set $E_{m} \in I$ such that

$$
\left\{n \in \mathbb{N}: \sum_{k=1}^{\infty} b_{n k}^{(i)} \chi_{A_{m}}(k) \geq \varepsilon_{m}\right\} \subseteq E_{m} \quad \forall i \in S
$$

and by (38) we can find a strictly increasing sequence $\left(M_{p}\right)_{p \in \mathbb{N}}$ in $\mathbb{N}$ such that

$$
\sup _{i \in S} \sup _{n \in B_{M_{p}}} \sum_{k=1}^{\infty} b_{n k}^{(i)} \chi_{\mathbb{N} \backslash B_{M_{p}}}(k) \leq \varepsilon_{p} \quad \forall p \in \mathbb{N} .
$$

Next we fix a strictly increasing sequence $\left(p_{m}\right)_{m \in \mathbb{N}}$ in $\mathbb{N}$ such that $E_{m} \subseteq B_{M_{p_{m}}}$ for every $m \in \mathbb{N}$. We write $F_{m}$ for $B_{M_{p_{m}}}$. Then $F_{m} \subseteq F_{m+1}$ and $\bigcup_{m=1}^{\infty} F_{m}=\mathbb{N}$.

Let $m(k)=\min \left\{m \in \mathbb{N}: k \in F_{m}\right\}$ for every $k \in \mathbb{N}$ and put

$$
t_{k}= \begin{cases}s_{k} & \text { if } k \notin A_{m(k)} \\ a & \text { if } k \in A_{m(k)}\end{cases}
$$

It is easily checked that $\left\{k \in \mathbb{N}:\left|t_{k}-a\right| \geq \varepsilon_{m}\right\} \subseteq F_{m}$ for every $m$ and hence $\left(t_{k}\right)_{k \in \mathbb{N}}$ is $I$-convergent to $a$.

Now it remains to show $C:=\left\{k \in \mathbb{N}: s_{k} \neq t_{k}\right\} \in J_{\mathscr{B}, I}$. To this end, fix $\varepsilon>0$ and choose $m$ such that $\sum_{l=m+1}^{\infty} \varepsilon_{l} \leq \varepsilon / 3$ and $\varepsilon_{p_{m}} \leq \varepsilon / 3$.

Since $I \subseteq J_{\mathscr{B}, I}$, we can find $E \in I$ with

$$
\left\{n \in \mathbb{N}: \sum_{k=1}^{\infty} b_{n k}^{(i)} \chi_{F_{m}}(k) \geq \frac{\varepsilon}{3}\right\} \subseteq E \quad \forall i \in S .
$$

Then $F_{m} \cup E \in I$ and for every $n \in \mathbb{N} \backslash\left(F_{m} \cup E\right)$ and each $i \in \mathrm{S}$ we have $m(n)>m$ and

$$
\begin{aligned}
& \sum_{k=1}^{\infty} b_{n k}^{(i)} \chi_{C}(k)=\sum_{k=1}^{\infty} b_{n k}^{(i)} \chi_{C \cap F_{m}}(k) \\
& +\sum_{k=1}^{\infty} b_{n k}^{(i)} \chi_{\operatorname{Cn}\left(\mathbb{N} \backslash F_{m}\right)}(k) \\
& \stackrel{(42)}{<} \frac{\varepsilon}{3}+\sum_{k=1}^{\infty} b_{n k}^{(i)} \chi_{\mathrm{C} \cap\left(\mathbb{N} \backslash F_{m(n)}\right)}(k) \\
& +\sum_{k=1}^{\infty} b_{n k}^{(i)} \chi_{C \cap\left(F_{m(n)} \backslash F_{m}\right)}(k) \\
& \stackrel{(40)}{\leq} \frac{\varepsilon}{3}+\varepsilon_{p_{m(n)}} \\
& +\sum_{l=m+1}^{m(n)} \sum_{k=1}^{\infty} b_{n k}^{(i)} \chi_{\mathrm{Cn}\left(F_{l} \backslash F_{l-1}\right)}(k) \\
& \leq \frac{\varepsilon}{3}+\varepsilon_{p_{m}}+\sum_{l=m+1}^{m(n)} \sum_{k=1}^{\infty} b_{n k}^{(i)} \chi_{A_{l}}(k) \\
& \stackrel{(39)}{\leq} \frac{2}{3} \varepsilon+\sum_{l=m+1}^{m(n)} \varepsilon_{l} \leq \varepsilon,
\end{aligned}
$$

which completes the proof.

Note that condition (38) is in particular satisfied for $B_{m}=$ $\{1, \ldots, m\}$ if $I=I_{f}$ and each $B_{i}$ is a lower triangular matrix.

Making use of his aforementioned characterisation of statistical convergence, Fridy further proved in [20] the 
following Tauberian theorem for statistical convergence: a statistically convergent sequence $\left(s_{n}\right)_{n \in \mathbb{N}}$ which satisfies $\mid s_{n}-$ $s_{n+1} \mid=O(1 / n)$ for $n \rightarrow \infty$ is convergent in the ordinary sense. It is not too difficult to obtain the following slightly more general result by modifying the proof from [20] accordingly (there the functions $\varphi, \psi$, and $h$ below are simply $\varphi(x)=1 / x=\psi(x)$ and $\left.h(x)=x(1+x)^{-1}\right)$. For the sake of brevity, we skip the details.

Theorem 12. Let $I$ be an admissible ideal and $A=\left(a_{n k}\right)_{n, k \geq 1}$ a lower triangular matrix such that I-lim $\sum_{k=1}^{n} a_{n k}=1$ and $I$ - $\lim a_{n k}=0$ for every $k \in \mathbb{N}$. Suppose that $\varphi, \psi$, and $h$ are functions from $[0, \infty)$ into itself such that $\varphi$ is decreasing on $(0, \infty), \min _{k=1, \ldots, n} a_{n k} \geq \psi(n)$ for every $n \in \mathbb{N}, I-\lim x_{n}=0$ whenever $I-\lim h\left(x_{n}\right)=0$, and

$$
x \psi(x+y) \geq h(x \varphi(y)) \quad \forall x, y \geq 0 .
$$

Let $\left(s_{n}\right)_{n \in \mathbb{N}}$ and $\left(t_{n}\right)_{n \in \mathbb{N}}$ be number sequences such that $\lim _{n \rightarrow \infty} t_{n}=0,\left\{n \in \mathbb{N}: s_{n} \neq t_{n}\right\} \in J_{A, I}$, and $\left|s_{n}-s_{n+1}\right|=$ $O(\varphi(n))$ for $n \rightarrow \infty$. Then I-lim $s_{n}=0$.

Combining Theorems 11 and 12 we get the following corollary.

Corollary 13. Under the same general hypothesis as in Theorem 12 with $I=I_{f}$, if $\left(s_{n}\right)_{n \in \mathbb{N}}$ is a sequence which is $A$ statistically convergent to the number $a$ and fulfills $\left|s_{n}-s_{n+1}\right|=$ $O(\varphi(n))$ for $n \rightarrow \infty$, then $\left(s_{n}\right)_{n \in \mathbb{N}}$ is convergent to $a$ in the usual sense.

\section{Limit Superior and Limit Inferior}

In [21] Demirci introduced the concepts of limit superior and limit inferior with respect to an ideal $I$ on $\mathbb{N}$, generalising the notions of statistical limit superior and limit inferior from [22]. For a sequence $\left(s_{n}\right)_{n \in \mathbb{N}}$ in $\mathbb{R}$ put

$$
\begin{aligned}
& I-\lim \sup s_{n}:=\sup \left\{t \in \mathbb{R}:\left\{n \in \mathbb{N}: s_{n}>t\right\} \notin I\right\}, \\
& I-\lim \inf s_{n}:=\inf \left\{t \in \mathbb{R}:\left\{n \in \mathbb{N}: s_{n}<t\right\} \notin I\right\} .
\end{aligned}
$$

The same definitions were independently introduced by the authors of [3]. Note that since $\left(s_{n}\right)_{n \in \mathbb{N}}$ is not assumed to be bounded, it can happen that these values are $\infty$ or $-\infty$. If $I=$ $I_{f}$ the above definitions are equivalent to the usual definitions of limit superior and limit inferior. It is proved in [21] (and in [3] as well) that $I-\lim \inf s_{n} \leq I-\lim \sup s_{n}$ and that $\left(s_{n}\right)_{n \in \mathbb{N}}$ is $I$-convergent to $a \in \mathbb{R}$ if and only if $I$-lim inf $s_{n}=a=$ $I$ - $\lim \sup _{n}$ (cf. [21, Theorems 3 and 4] or [3, Theorems 3.2 and 3.4]).

Let us also remark that

$$
\begin{aligned}
& I-\lim \sup s_{n}=\inf _{A \in I} \sup \left\{s_{n}: n \in \mathbb{N} \backslash A\right\}, \\
& I-\lim \inf s_{n}=\sup _{A \in I} \inf \left\{s_{n}: n \in \mathbb{N} \backslash A\right\},
\end{aligned}
$$

as is easily checked.

In [22, Lemma on p.3628] necessary and sufficient conditions for a real matrix $A$ to satisfy the inequality lim sup $A x \leq$ st-lim $\sup x$ for all $x \in \ell^{\infty}$ were obtained (here, st-limsup $x$ denotes the aforementioned statistical limit superior that was introduced in [22]; in our terminology it is nothing but the limit superior with respect to the ideal $J_{C, I_{f}}$, where $C$ is the Cesàro matrix).

Later, Demirci gave a more general necessity result concerning the $I$-limit superior and the $I$-limit inferior (cf. [21, Corollary 1]). The following proposition is a further generalisation of this result while its proof follows the lines from [22].

Proposition 14. Let $I, J$ be ideals on $\mathbb{N}$ and $A=\left(a_{n k}\right)_{n, k \in \mathbb{N}}$ an infinite matrix in $\mathbb{R}$ such that the following conditions are satisfied:

$$
\begin{gathered}
\sum_{k=1}^{\infty}\left|a_{n k}\right|<\infty \quad \forall n \in \mathbb{N}, \\
I-\lim \sum_{k=1}^{\infty}\left|a_{n k}\right|=1=I-\lim \sum_{k=1}^{\infty} a_{n k}, \\
I-\lim \sum_{k=1}^{\infty}\left|a_{n k}\right| \chi_{E}(k)=0 \quad \forall E \in J .
\end{gathered}
$$

Then

$$
I-\lim \sup A s \leq J-\lim \sup s \quad \forall s \in \ell^{\infty}
$$

as well as

$$
I-\lim \inf A s \geq J-\liminf s \quad \forall s \in \ell^{\infty} .
$$

Proof. Let $s=\left(s_{n}\right)_{n \in \mathbb{N}} \in \ell^{\infty}$ be arbitrary and put $b=$ $J$-limsups. Since $s$ is bounded, we have $b \in \mathbb{R}$. Also, fix an arbitrary $\varepsilon>0$. Then by [21, Theorem 1] (or [3, Theorem 3.1]) we have $E:=\left\{n \in \mathbb{N}: s_{n}>b+\varepsilon\right\} \in J$. We put $F=\mathbb{N} \backslash E$.

For every $a \in \mathbb{R}$ set $a^{+}=\max \{a, 0\}$ and $a^{-}=\max \{-a, 0\}$, as in [22]. Note that $a=a^{+}-a^{+}$and $|a|=a^{+}+a^{-}$.

Then for every $n \in \mathbb{N}$

$$
\begin{aligned}
(A s)(n)= & \sum_{k=1}^{\infty} a_{n k} s_{k}=\sum_{k=1}^{\infty} a_{n k}^{+} \chi_{E}(k) s_{k} \\
& +\sum_{k=1}^{\infty} a_{n k}^{+} \chi_{F}(k) s_{k}-\sum_{k=1}^{\infty} a_{n k}^{-} s_{k} \\
\leq & \|s\|_{\infty} \sum_{k=1}^{\infty}\left|a_{n k}\right| \chi_{E}(k)+(b+\varepsilon) \sum_{k=1}^{\infty} a_{n k}^{+} \chi_{F}(k) \\
& +\frac{1}{2}\|s\|_{\infty} \sum_{k=1}^{\infty}\left(\left|a_{n k}\right|-a_{n k}\right) \\
= & \|s\|_{\infty} \sum_{k=1}^{\infty}\left|a_{n k}\right| \chi_{E}(k)+\frac{1}{2}\|s\|_{\infty} \sum_{k=1}^{\infty}\left(\left|a_{n k}\right|-a_{n k}\right) \\
& +\frac{b+\varepsilon}{2}\left(\sum_{k=1}^{\infty}\left(\left|a_{n k}\right|+a_{n k}\right)\left(1-\chi_{E}(k)\right)\right) .
\end{aligned}
$$


Because of $E \in J$ and the assumptions (48) and (49) the $I$ limit of the right-hand side of the above inequality is equal to $b+\varepsilon$. Together with the obvious monotonicity of $I$-lim sup it follows that $I-\lim \sup A s \leq b+\varepsilon$. Since $\varepsilon>0$ was arbitrary, the proof is finished.

The second statement follows from the first one by multiplication with -1 .

It was also proved in [22] that a sequence of real numbers which is bounded above and Cesàro-summable to its statistical limit superior is statistically convergent (cf. [22, Theorem 5]). It is possible to modify the proof of [22] to obtain the following more general result. We use the same notation as in the previous section.

Theorem 15. Suppose that each $B_{i}$ is nonnegative, $\sum_{k=1}^{\infty} b_{n k}^{(i)}<$ $\infty$ for all $n \in \mathbb{N}, i \in S$, and

$$
I-\lim \sum_{k=1}^{\infty} b_{n k}^{(i)}=1 \quad \text { uniformly in } i \in S \text {. }
$$

If $s=\left(s_{n}\right)_{n \in \mathbb{N}}$ is a bounded sequence of real numbers and $a \in \mathbb{R}$ such that $s$ is $\mathscr{B}^{I}$-summable to a and $J_{\mathscr{B}, I}$-lim sup $s=$ a or $J_{\mathscr{B}, I^{-}}$ $\lim \inf s=a$, then $s$ is $\mathscr{B}^{I}$-statistically convergent to a.

Proof. It is enough to prove the statement for the case $J_{\mathscr{B}, I^{-}}$ $\lim \sup s=a$. Suppose that $s$ is not $B^{I}$-statistically convergent to $a$. Then $J_{\mathscr{B}, I}-\lim \inf s<a$ and hence there must be some $t<a$ such that $E:=\left\{n \in \mathbb{N}: s_{n}<t\right\} \notin J_{\mathscr{B}, I}$. Consequently, there exists a $d>0$ such that

$$
A:=\left\{n \in \mathbb{N}: \sup _{i \in S} \sum_{k=1}^{\infty} b_{n k}^{(i)} \chi_{E}(k) \geq d\right\} \notin I .
$$

Fix an arbitrary $\varepsilon>0$ and put $F:=\left\{n \in \mathbb{N}: t \leq s_{n} \leq a+\varepsilon\right\}$ and $G:=\left\{n \in \mathbb{N}: s_{n}>a+\varepsilon\right\}$. Take $\delta \in(0, \varepsilon)$ with $\delta|a+\varepsilon| \leq \varepsilon$. By our assumption (53) we have

$$
C:=\left\{n \in \mathbb{N}: \sup _{i \in S}\left|\sum_{k=1}^{\infty} b_{n k}^{(i)}-1\right| \geq \delta\right\} \in I .
$$

It follows from [21, Theorem 1] that $G \in J_{\mathscr{B}, I}$ and hence

$$
D:=\left\{n \in \mathbb{N}: \sup _{i \in S} \sum_{k=1}^{\infty} b_{n k}^{(i)} \chi_{G}(k) \geq \delta\right\} \in I .
$$

Now let $n \in H:=A \cap(\mathbb{N} \backslash(C \cup D))$ be arbitrary. Since $n \in A$, there is some $i \in S$ such that $\sum_{k=1}^{\infty} b_{n k}^{(i)} \chi_{E}(k)>d / 2$. Write
$M=\|s\|_{\infty}$. It then follows from the definitions of the sets $E, F, G, C$, and $D$ and the choice of $\delta$ that

$$
\begin{aligned}
& \sum_{k=1}^{\infty} b_{n k}^{(i)} s_{k}=\sum_{k=1}^{\infty} b_{n k}^{(i)} s_{k} \chi_{E}(k) \\
& +\sum_{k=1}^{\infty} b_{n k}^{(i)} s_{k} \chi_{F}(k)+\sum_{k=1}^{\infty} b_{n k}^{(i)} s_{k} \chi_{G}(k) \\
& \leq t \sum_{k=1}^{\infty} b_{n k}^{(i)} \chi_{E}(k) \\
& +(a+\varepsilon) \sum_{k=1}^{\infty} b_{n k}^{(i)} \chi_{F}(k)+M \delta \\
& =t \sum_{k=1}^{\infty} b_{n k}^{(i)} \chi_{E}(k)+M \delta \\
& +(a+\varepsilon) \sum_{k=1}^{\infty} b_{n k}^{(i)}\left(1-\chi_{E}(k)-\chi_{G}(k)\right) \\
& \leq t \sum_{k=1}^{\infty} b_{n k}^{(i)} \chi_{E}(k)+M \delta \\
& +(a+\varepsilon)\left(1-\sum_{k=1}^{\infty} b_{n k}^{(i)} \chi_{E}(k)\right) \\
& +|a+\varepsilon|\left(\sum_{k=1}^{\infty} b_{n k}^{(i)} \chi_{G}(k)+\left|\sum_{k=1}^{\infty} b_{n k}^{(i)}-1\right|\right) \\
& \leq a+\varepsilon+M \varepsilon+(t-a-\varepsilon) \\
& \times \sum_{k=1}^{\infty} b_{n k}^{(i)} \chi_{E}(k)+2|a+\varepsilon| \delta \\
& <a+\varepsilon(M+3)+(t-a-\varepsilon) \frac{d}{2} \text {. }
\end{aligned}
$$

Thus we have

$$
\begin{aligned}
\sup _{i \in S} \mid & a-\sum_{k=1}^{\infty} b_{n k}^{(i)} s_{k} \mid \\
& >\frac{d}{2}(a+\varepsilon-t)-\varepsilon(M+3) \quad \forall n \in H .
\end{aligned}
$$

Suppose that

$$
\begin{aligned}
h:=I-\lim \sup \sup _{i \in S}\left|a-\sum_{k=1}^{\infty} b_{n k}^{(i)} s_{k}\right| \\
\quad<\frac{d}{2}(a+\varepsilon-t)-\varepsilon(M+3) .
\end{aligned}
$$

Then it would follow that $H \in I$. But $C, D \in I$ and hence

$$
A=H \cup(C \cap A) \cup(D \cap A) \in I,
$$

contradicting (54). 
Thus $h \geq(d / 2)(a+\varepsilon-t)-\varepsilon(M+3)$ and since $\varepsilon>0$ was arbitrary, we get $h \geq(a-t) d / 2>0$ and hence $s$ is not $B^{I}$-summable to $a$.

We conclude this section with a lemma that will be needed later and may also be of independent interest. First we need one more definition: a number sequence $\left(s_{n}\right)_{n \in \mathbb{N}}$ is called $I$-bounded if there is a constant $K>0$ such that $\left\{n \in \mathbb{N}:\left|s_{n}\right|>K\right\} \in I$. Note that $I$-convergent sequences are $I$-bounded and that the $I$-boundedness of $\left(s_{n}\right)_{n \in \mathbb{N}}$ implies that $I$-lim $\sup s_{n}$ and $I$-lim inf $s_{n}$ are finite.

Lemma 16. For any ideal I on $\mathbb{N}$ and all I-bounded sequences $\left(s_{n}\right)_{n \in \mathbb{N}}$ and $\left(t_{n}\right)_{n \in \mathbb{N}}$ in $\mathbb{R}$ the inequalities

$$
\begin{aligned}
& I-\lim \sup \left(s_{n}+t_{n}\right) \leq I-\lim \sup s_{n}+I-\lim \sup t_{n}, \\
& I-\lim \sup \left(s_{n}+t_{n}\right) \geq I-\lim \sup s_{n}+I-\lim \sup t_{n}
\end{aligned}
$$

hold. If one of the sequences is I-convergent, then equality holds.

Proof. It is enough to prove the statement for the $I$-lim sup. Let $a=I-\lim \sup s_{n}$ and $b=I$-limsupt $t_{n}$. If $u, v \in \mathbb{R}$ such that $u>a$ and $v>b$ then $A:=\left\{n \in \mathbb{N}: s_{n}>u\right\} \in I$ and $B:=\left\{n \in \mathbb{N}: t_{n}>v\right\} \in I$. Hence $A \cup B \in I$. But

$$
C:=\left\{n \in \mathbb{N}: s_{n}+t_{n}>u+v\right\} \subseteq A \cup B,
$$

and thus $C \in I$.

If $I-\lim \sup \left(s_{n}+t_{n}\right)>u+v$, then there would be some $\eta>u+v$ such that $\left\{n \in \mathbb{N}: s_{n}+t_{n}>\eta\right\} \notin I$, which would imply $C \notin I$. Thus we must have $I$-lim $\sup \left(s_{n}+t_{n}\right) \leq$ $u+v$. Since $u>a$ and $v>b$ were arbitrary, it follows that $I-\lim \sup \left(s_{n}+t_{n}\right) \leq a+b$.

Now suppose that $\left(s_{n}\right)_{n \in \mathbb{N}}$ is $I$-convergent to $a$ and fix an arbitrary $\varepsilon>0$. Put $D:=\left\{n \in \mathbb{N}: s_{n}+t_{n}>a+b-\varepsilon\right\}$, $E:=\left\{n \in \mathbb{N}: s_{n}>a-\varepsilon / 2\right\}$, and $F:=\left\{n \in \mathbb{N}: t_{n}>b-\varepsilon / 2\right\}$.

By [21, Theorem 1] $F \notin I$ and because of $I-\lim s_{n}=a$ we have $\mathbb{N} \backslash E \in I$, that is, $E \in \mathscr{F}(I)$.

If $E \cap F \in I$, then $(\mathbb{N} \backslash E) \cup(\mathbb{N} \backslash F) \in \mathscr{F}(I)$ and hence $(\mathbb{N} \backslash F) \cap E=((\mathbb{N} \backslash E) \cup(\mathbb{N} \backslash F)) \cap E \in \mathscr{F}(I)$; thus $\mathbb{N} \backslash F \in \mathscr{F}(I)$, contradicting the fact that $F \notin I$.

So we must have $E \cap F \notin I$ and since $E \cap F \subseteq D$, it follows that $D \notin I$, which implies $I$-lim $\sup \left(s_{n}+t_{n}\right) \geq a+b-\varepsilon$. Letting $\varepsilon \rightarrow 0$ completes the proof.

\section{Cluster Points}

Fridy [23] defined and studied statistical cluster points and statistical limit points of a sequence. These concepts were later generalised by the authors of [1] to an arbitrary admissible ideal $I$. Consider a sequence $\left(x_{n}\right)_{n \in \mathbb{N}}$ in a metric space $(X, d)$. An element $x \in X$ is called an $I$-cluster point of $\left(x_{n}\right)_{n \in \mathbb{N}}$ if $\left\{n \in \mathbb{N}: d\left(x_{n}, x\right)<\varepsilon\right\} \notin I$ for every $\varepsilon>0$ and it is called an $I$-limit point of $\left(x_{n}\right)_{n \in \mathbb{N}}$ if there is a subsequence $\left(x_{n_{k}}\right)_{k \in \mathbb{N}}$ with $\left\{n_{k}: k \in \mathbb{N}\right\} \notin I$ that converges to $x$. For $I=I_{f}$, both notions are equivalent to the usual notion of cluster points. Every $I$-limit point is also an $I$-cluster point of $\left(x_{n}\right)_{n \in \mathbb{N}}$ (cf. [1, Proposition 4.1]) but the converse is not true in general. It was shown in [3, Theorem 3.5] that a bounded sequence $\left(s_{n}\right)_{n \in \mathbb{N}}$ in $\mathbb{R}$ always possesses an $I$-cluster point and that the $I$ lim sup and the $I$-lim inf of the sequence are the greatest and the smallest of them, respectively. It is easily observed that the same proof still works if the sequence is only $I$-bounded.

Concerning $J_{\mathscr{B}, I}$-cluster points, we can give the following characterisation.

Proposition 17. Suppose that $\sup _{n \in \mathbb{N}, i \in S} \sum_{k=1}^{\infty} b_{n k}^{(i)}<\infty$ and

$$
I-\lim \sum_{k=1}^{\infty} b_{n k}^{(i)}=1 \quad \text { uniformlyini } \in \text { S. }
$$

Then $a$ is a $J_{\mathscr{B}, I}$-cluster point of $s=\left(s_{n}\right)_{n \in \mathbb{N}}$ if and only if for every $\varepsilon>0$

$$
I-\lim \inf \inf _{i \in S} \sum_{k=1}^{\infty} b_{n k}^{(i)} \chi_{D(s, a, \varepsilon)}(k)<1 .
$$

Proof. Put $A_{\varepsilon}=D(s, a, \varepsilon)$ and $B_{\varepsilon}=\mathbb{N} \backslash A_{\varepsilon}$ for every $\varepsilon>0$. By definition, $a$ is a $J_{\mathscr{B}, I}$-cluster point of $s$ if and only if $B_{\varepsilon} \notin J_{\mathscr{B}, I}$ for every $\varepsilon>0$ which is the case if and only if

$$
I-\lim \sup \sup _{i \in S} \sum_{k=1}^{\infty} b_{n k}^{(i)} \chi_{B_{\varepsilon}}(k)>0 .
$$

But $\sum_{k=1}^{\infty} b_{n k}^{(i)} \chi_{B_{\varepsilon}}(k)=\sum_{k=1}^{\infty} b_{n k}^{(i)}-\sum_{k=1}^{\infty} b_{n k}^{(i)} \chi_{A_{\varepsilon}}(k)$, so because of (63) and Lemma 16 it follows that $a$ is a $J_{\mathscr{B}, I}$-cluster point of $s$ if and only if

$$
\begin{aligned}
I-\lim \sup & \sup _{i \in S}\left(1-\sum_{k=1}^{\infty} b_{n k}^{(i)} \chi_{A_{\varepsilon}}(k)\right)>0 \\
& \Longleftrightarrow 1-I \text {-lim inf } \inf _{i \in S} \sum_{k=1}^{\infty} b_{n k}^{(i)} \chi_{A_{\varepsilon}}(k)>0
\end{aligned}
$$

and the proof is finished.

This characterisation yields the following sufficient condition for a $J_{\mathscr{B}, I}$-cluster point.

Corollary 18. Under the same assumptions as in the previous proposition, if $\mathscr{F}=\left(F_{k}^{(i)}\right)_{k \in \mathbb{N}, i \in S}$ is a family in $\mathscr{M} \cup \mathcal{O}$ such that

$$
\begin{gathered}
L(t):=\inf \left\{F_{k}^{(i)}(t): k \in \mathbb{N}, i \in S\right\}>0 \quad \forall t>0, \\
I \text {-liminf } \inf _{i \in S} \sum_{k=1}^{\infty} b_{n k}^{(i)} F_{k}^{(i)}\left(\left|s_{k}-a\right|\right)=0,
\end{gathered}
$$

then $a$ is a $J_{\mathscr{B}, I^{-}}$-cluster point of $s$.

Proof. For every $\varepsilon>0$ and all $i \in S, n \in \mathbb{N}$ we have

$$
\begin{aligned}
& \sum_{k=1}^{\infty} b_{n k}^{(i)} F_{k}^{(i)}\left(\left|s_{k}-a\right|\right) \\
& \quad \geq \sum_{k=1}^{\infty} b_{n k}^{(i)} F_{k}^{(i)}\left(\left|s_{k}-a\right|\right) \chi_{D(s, a, \varepsilon)}(k) \\
& \quad \geq L(\varepsilon) \sum_{k=1}^{\infty} b_{n k}^{(i)} \chi_{D(s, a, \varepsilon)}(k)
\end{aligned}
$$


and thus it follows from the assumptions that

$$
I-\lim \inf \inf _{i \in S} \sum_{k=1}^{\infty} b_{n k}^{(i)} \chi_{D(s, a, \varepsilon)}(k)=0<1 \quad \forall \varepsilon>0 .
$$

Hence by the previous proposition, $a$ is a $J_{\mathscr{B}, I}$-cluster point of $s$.

\section{Pre-Cauchy Sequences}

The authors of [24] introduced the notion of statistically pre-Cauchy sequences. The sequence $s=\left(s_{k}\right)_{k \in \mathbb{N}}$ is called a statistically pre-Cauchy sequence if $\lim _{n \rightarrow \infty} 1 / n^{2} \mid\{(i, j) \in$ $\left.\{1, \ldots, n\}^{2}:\left|s_{i}-s_{j}\right| \geq \varepsilon\right\} \mid=0$ for every $\varepsilon>0$. They show that a statistically convergent sequence is statistically preCauchy and that the converse is not true in general but under certain additional assumptions. It is further proved that $s$ is statistically pre-Cauchy if

$$
\lim _{n \rightarrow \infty} \frac{1}{n^{2}} \sum_{i=1}^{n} \sum_{j=1}^{n}\left|s_{i}-s_{j}\right|=0
$$

and that the converse is true if $s$ is bounded (cf. [24, Theorem 3]).

We propose the following generalisation of the definition of statistically pre-Cauchy sequences to our setting.

Definition 19. If each $B_{i}$ is nonnegative, a sequence $s=\left(s_{k}\right)_{k \in \mathbb{N}}$ of real or complex numbers is called a $\mathscr{B}^{I}$-statistically preCauchy sequence if for every $\varepsilon>0$

$$
I-\lim \sum_{k=1}^{\infty} \sum_{l=1}^{\infty} b_{n k}^{(i)} b_{n l}^{(i)} \chi_{D(s, \varepsilon)}(k, l)=0 \quad \text { uniformly in } i \in S \text {, }
$$

where $D(s, \varepsilon):=\left\{(k, l) \in \mathbb{N}^{2}:\left|s_{k}-s_{l}\right| \geq \varepsilon\right\}$.

First we show that, under an additional assumption on $\mathscr{B}, \mathscr{B}^{I}$-statistically convergent sequences are $\mathscr{B}^{I}$-statistically pre-Cauchy.

Lemma 20. Suppose that $s$ is $\mathscr{B}^{I}$-statistically convergent and

$$
\exists A \in I M:=\sup \left\{\sum_{k=1}^{\infty} b_{n k}^{(i)}: n \in \mathbb{N} \backslash A, i \in S\right\}<\infty .
$$

Then s is a $\mathscr{B}^{I}$-statistically pre-Cauchy sequence.

Proof. Say $s$ is $\mathscr{B}^{I}$-statistically convergent to $a$. For every $\varepsilon>0$ and all $n \in \mathbb{N} \backslash A$ we have

$$
\begin{aligned}
& \sum_{k=1}^{\infty} \sum_{l=1}^{\infty} b_{n k}^{(i)} b_{n l}^{(i)} \chi_{D(s, \varepsilon)}(k, l) \\
& \leq \sum_{k=1}^{\infty} \sum_{l=1}^{\infty} b_{n k}^{(i)} b_{n l}^{(i)}\left(\chi_{D(s, a, \varepsilon / 2)}(k)+\chi_{D(s, a, \varepsilon / 2)}(l)\right) \\
& \leq 2 M \sum_{k=1}^{\infty} b_{n k}^{(i)} \chi_{D(s, a, \varepsilon / 2)}(k) \longrightarrow 0 \quad \text { along } I \text { uniformly in } i \in S .
\end{aligned}
$$

The next two propositions are the analogues of [24, Theorem 3]. Since their proofs parallel very much those of Proposition 3 and Proposition 4, respectively, they will be omitted. In the formulation of both propositions, we differ from our usual notation and allow $\mathscr{F}=\left(F_{k l}^{(i)}\right)_{k, l \in \mathbb{N}, i \in S}$ to be a family in $\mathscr{M} \cup \mathcal{O}$ with index set $\mathbb{N} \times \mathbb{N} \times S$ instead of $\mathbb{N} \times S$.

Proposition 21. Suppose that

$$
\begin{gathered}
I-\lim \sum_{k=1}^{\infty} \sum_{l=1}^{\infty} b_{n k}^{(i)} b_{n l}^{(i)} F_{k l}^{(i)}\left(\left|s_{k}-s_{l}\right|\right)=0 \quad \text { uniformlyin } i \in S, \\
L(t):=\inf \left\{F_{k l}^{(i)}(t): k, l \in \mathbb{N}, i \in S\right\}>0 \quad \forall t>0 .
\end{gathered}
$$

Then s is $\mathscr{B}^{I}$-statistically pre-Cauchy.

Proposition 22. Suppose that $s$ is bounded and $\mathscr{B}^{I}$-statistically pre-Cauchy. If $\mathscr{F}$ is equicontinuous at 0 and

$$
\exists A \in I M:=\sup \left\{\sum_{k=1}^{\infty} b_{n k}^{(i)}: n \in \mathbb{N} \backslash A, i \in S\right\}<\infty,
$$

as well as

$$
h(t):=\sup \left\{F_{k l}^{(i)}(t): k, l \in \mathbb{N}, i \in S\right\}<\infty \quad \forall t \geq 0,
$$

then we also have

$$
I-\lim \sum_{k=1}^{\infty} \sum_{l=1}^{\infty} b_{n k}^{(i)} b_{n l}^{(i)} F_{k l}^{(i)}\left(\left|s_{k}-s_{l}\right|\right)=0 \quad \text { uniformly in } i \in S .
$$

It was proved in [24] that a statistically pre-Cauchy sequence $\left(s_{n}\right)_{n \in \mathbb{N}}$ which possesses a convergent subsequence $\left(s_{n_{k}}\right)_{k \in \mathbb{N}}$ such that the set of indices $\left\{n_{k}: k \in \mathbb{N}\right\}$ is "large" in the sense that

$$
\liminf _{n \rightarrow \infty} \frac{1}{n}\left|\left\{n_{k}: k \in \mathbb{N}, n_{k} \leq n\right\}\right|>0
$$

is statistically convergent. This result can be generalised in the following way.

Theorem 23. Suppose that $I \subseteq J_{\mathscr{B}, I}$ and

$$
\sup \left\{\sum_{k=1}^{\infty} b_{n k}^{(i)}: n \in \mathbb{N}, i \in S\right\}<\infty \text {. }
$$

Let a be any real or complex number. Let $s=\left(s_{n}\right)_{n \in \mathbb{N}}$ be a $\mathscr{B}^{I}$ statistically pre-Cauchy sequence and let $W \subseteq \mathbb{N}$ be such that for every $\varepsilon>0$ the set $\left\{n \in W:\left|s_{n}-a\right| \geq \varepsilon\right\}$ belongs to $I$ and furthermore

$$
w:=I-\lim \inf \inf _{i \in S} \sum_{k=1}^{\infty} b_{n k}^{(i)} \chi_{W}(k)>0 .
$$

Then s is $\mathscr{B}^{I}$-statistically convergent to a. 
Proof. Take $\varepsilon, \delta>0$ arbitrary. Then $V:=\left\{k \in W:\left|s_{k}-a\right| \geq\right.$ $\varepsilon / 2\} \in I$, by assumption. Put $A:=\left\{k \in W:\left|s_{k}-a\right|<\varepsilon / 2\right\}$, $B:=\left\{k \in \mathbb{N}:\left|s_{k}-a\right| \geq \varepsilon\right\}$, and $C:=\left\{(k, l) \in \mathbb{N}^{2}:\left|s_{k}-s_{l}\right| \geq\right.$ $\varepsilon / 2\}$. Then $A \times B \subseteq C$.

Let us also fix $\tau \in(0, w)$ such that $\tau(w-\tau)^{-1} \leq \delta$. Since $s$ is $\mathscr{B}^{I}$-statistically pre-Cauchy, there is some $E \in I$ such that

$$
\left\{n \in \mathbb{N}: \sum_{k=1}^{\infty} \sum_{l=1}^{\infty} b_{n k}^{(i)} b_{n l}^{(i)} \chi_{C}(k, l) \geq \tau\right\} \subseteq E \quad \forall i \in S .
$$

But we have

$$
\begin{aligned}
& \sum_{k=1}^{\infty} \sum_{l=1}^{\infty} b_{n k}^{(i)} b_{n l}^{(i)} \chi_{C}(k, l) \\
& \quad \geq\left(\sum_{k=1}^{\infty} b_{n k}^{(i)} \chi_{A}(k)\right)\left(\sum_{l=1}^{\infty} b_{n l}^{(i)} \chi_{B}(l)\right)
\end{aligned}
$$

and thus

$$
\left\{n \in \mathbb{N}:\left(\sum_{k=1}^{\infty} b_{n k}^{(i)} \chi_{A}(k)\right)\left(\sum_{l=1}^{\infty} b_{n l}^{(i)} \chi_{B}(l)\right) \geq \tau\right\} \subseteq E \quad \forall i \in S .
$$

Since $V \in I \subseteq J_{\mathscr{B}, I}$, it follows that

$$
I-\lim \sup _{i \in S} \sum_{k=1}^{\infty} b_{n k}^{(i)} \chi_{V}(k)=0 .
$$

Because of Lemma 16 this implies

$$
\begin{aligned}
w & =I-\lim \inf \inf _{i \in S} \sum_{k=1}^{\infty} b_{n k}^{(i)}\left(\chi_{A}(k)+\chi_{V}(k)\right) \\
& \leq I-\lim \inf \left(\inf _{i \in S} \sum_{k=1}^{\infty} b_{n k}^{(i)} \chi_{A}(k)+\sup _{i \in S} \sum_{k=1}^{\infty} b_{n k}^{(i)} \chi_{V}(k)\right) \\
& =I-\lim \inf \inf _{i \in S} \sum_{k=1}^{\infty} b_{n k}^{(i)} \chi_{A}(k)=: r .
\end{aligned}
$$

By [21, Theorem 2] we have

$$
F:=\left\{n \in \mathbb{N}: \inf _{i \in S} \sum_{k=1}^{\infty} b_{n k}^{(i)} \chi_{A}(k)<r-\tau\right\} \in I .
$$

If $n \in \mathbb{N} \backslash(E \cup F)$, then $\sum_{k=1}^{\infty} b_{n k}^{(i)} \chi_{B}(k)<\tau(r-\tau)^{-1} \leq \tau(w-$ $\tau)^{-1} \leq \delta$ for every $i \in S$.

Thus $E \cup F \in I$ with

$$
\left\{n \in \mathbb{N}: \sum_{k=1}^{\infty} b_{n k}^{(i)} \chi_{B}(k) \geq \delta\right\} \subseteq E \cup F \quad \forall i \in S
$$

and the proof is finished.

By [24, Theorem 5] a bounded statistically pre-Cauchy sequence in $\mathbb{R}$ whose set of cluster points is nowhere dense is statistically convergent. To obtain an analogous result in our setting, we introduce the following strengthening of the notion of $\mathscr{B}^{I}$-statistically pre-Cauchy sequences.
Definition 24. If each $B_{i}$ is nonnegative, a sequence $s=$ $\left(s_{k}\right)_{k \in \mathbb{N}}$ of real or complex numbers is called a $\mathscr{B}_{+}^{I}$-statistically pre-Cauchy sequence if for every $\varepsilon>0$

$$
I-\lim \sum_{k=1}^{\infty} \sum_{l=1}^{\infty} b_{n k}^{(i)} b_{n l}^{(j)} \chi_{D(s, \varepsilon)}(k, l)=0 \quad \text { uniformly in } i, j \in S .
$$

For $\mathscr{B}_{+}^{I}$-statistically pre-Cauchy sequences, Lemma 20, Proposition 21, and Proposition 22 hold accordingly (with the obvious modifications, one can even take a family $\mathscr{F}=$ $\left(F_{k l}^{(i, j)}\right)_{k, l \in \mathbb{N}, i, j \in S}$ in $\mathscr{M} \cup \mathcal{O}$ with index set $\mathbb{N}^{2} \times S^{2}$ in this case).

The next lemma generalises [24, Lemma 4] while its proof follows the same lines.

Lemma 25. Let $I$ be an admissible ideal. Suppose that $\sum_{k=1}^{\infty} b_{n k}^{(i)}<\infty$ for all $n \in \mathbb{N}, i \in S$ and

$$
\begin{gathered}
\exists A \in I \quad M:=\sup \left\{\sum_{k=1}^{\infty} b_{n k}^{(i)}: n \in \mathbb{N} \backslash A, i \in S\right\}<\infty, \\
I-\lim \sum_{k=1}^{\infty} b_{n k}^{(i)}=1 \quad \text { uniformlyini } \in S .
\end{gathered}
$$

Let $\mathscr{W}$ be a basis for $\mathscr{F}(I)$ such that for every $\left\{n_{1}<n_{2} \cdots n_{k}<\right.$ $\left.n_{k+1} \cdots\right\} \in \mathscr{W}$ the following holds:

$$
\exists k_{0} \in \mathbb{N}, \forall k \geq k_{0} \inf _{i \in S} \sum_{l=1}^{\infty}\left|b_{n_{k} l}^{(i)}-b_{n_{k+1} l}^{(i)}\right|<\frac{1}{3} .
$$

Let $s=\left(s_{n}\right)_{n \in \mathbb{N}}$ be a $\mathscr{B}_{+}^{I}$-statistically pre-Cauchy sequence in $\mathbb{R}$ and $\alpha<\beta$ such that $H:=\left\{n \in \mathbb{N}: s_{n} \in(\alpha, \beta)\right\} \in J_{\mathscr{B}, I}$.

Then $X:=\left\{n \in \mathbb{N}: s_{n} \leq \alpha\right\} \in J_{\mathscr{B}, I}$ or $Y:=\left\{n \in \mathbb{N}: s_{n} \geq\right.$ $\beta\} \in J_{\mathscr{B}, I}$.

Proof. Let us put $t_{n}=s_{n}$ if $n \notin H$ and $t_{n}=\alpha$ if $n \in H$. Since $H \in J_{\mathscr{B}, I}$, it is not difficult to see that $t=\left(t_{n}\right)_{n \in \mathbb{N}}$ is also $\mathscr{B}_{+}^{I}$-statistically pre-Cauchy. Put $P:=\left\{n \in \mathbb{N}: t_{n} \leq \alpha\right\}$ and $Q:=\left\{n \in \mathbb{N}: t_{n} \geq \beta\right\}$. Then $X \subseteq P \cup H$ and $Y \subseteq Q \cup H$; thus it suffices to show $P \in J_{\mathscr{B}, I}$ or $Q \in J_{\mathscr{B}, I}$. Note also that $t_{n} \notin(\alpha, \beta)$ for all $n \in \mathbb{N}$ and hence $Q=\mathbb{N} \backslash P$.

For the sake of brevity, we define for $n \in \mathbb{N}$ and $i \in S$

$$
D_{n i}(K):=\sum_{k=1}^{\infty} b_{n k}^{(i)} \chi_{A}(k) \quad \forall K \subseteq \mathbb{N} .
$$

We claim that

$$
I-\lim D_{n i}(P)\left(1-D_{n j}(P)\right)=0 \quad \text { uniformly in } i, j \in S \text {. }
$$

To see this, fix an arbitrary $\varepsilon>0$ and note that $P \times Q \subseteq$ $D(t, \beta-\alpha)$. So, since $t$ is $\mathscr{B}_{+}^{I}$-statistically pre-Cauchy, there is some $E \in I$ such that

$$
\left\{n \in \mathbb{N}: D_{n i}(P) D_{n j}(Q) \geq \frac{\varepsilon}{2}\right\} \subseteq E \quad \forall i, j \in S .
$$

By (90) there exists $F \in I$ such that

$$
\left\{n \in \mathbb{N}:\left|D_{n i}(\mathbb{N})-1\right| \geq \frac{\varepsilon}{2 M}\right\} \subseteq F \quad \forall i \in S
$$


Because of $(89)$ and $D_{n i}(Q)=D_{n i}(\mathbb{N})-D_{n i}(P)$ this easily implies

$$
\left\{n \in \mathbb{N}:\left|D_{n i}(P)\left(1-D_{n j}(P)\right)\right| \geq \varepsilon\right\} \subseteq E \cup F \cup A \quad \forall i, j \in S,
$$

proving our claim. In particular, we can find $C \in \mathscr{W}$ with

$$
\left|D_{n i}(P)\left(1-D_{n j}(P)\right)\right|<\frac{1}{9} \quad \forall n \in C, \forall i, j \in S .
$$

Then for every $n \in C$ we must have

$$
\sup _{i \in S} D_{n i}(P) \leq \frac{1}{3} \quad \text { or } \quad \inf _{j \in S} D_{n j}(P) \geq \frac{2}{3} .
$$

Write $C=\left\{n_{1}<n_{2} \cdots n_{k}<n_{k+1} \cdots\right\}$ and choose $k_{0}$ according to (91). Suppose first that $\sup _{i \in S} D_{n_{k_{0}} i}(P) \leq 1 / 3$. Then the same must hold for every $k>k_{0}$; for elsewise we could find a minimal $k>k_{0}$ with $\inf _{i \in S} D_{n_{k} i}(P) \geq 2 / 3$ which would imply

$$
\sum_{l=1}^{\infty}\left|b_{n_{k} l}^{(i)}-b_{n_{k-1} l}^{(i)}\right| \geq D_{n_{k} i}(P)-D_{n_{k-1} i}(P) \geq \frac{2}{3}-\frac{1}{3}=\frac{1}{3}
$$

for all $i \in S$, contradicting the choice of $k_{0}$.

So we have $D_{n_{k} i}(P) \leq 1 / 3$ for all $k \geq k_{0}$ and all $i \in S$. Now fix again an arbitrary $\varepsilon>0$. By (93) there is $G \in I$ such that

$$
\left\{n \in \mathbb{N}:\left|D_{n i}(P)\left(1-D_{n j}(P)\right)\right| \geq \frac{2}{3} \varepsilon\right\} \subseteq G \quad \forall i, j \in S .
$$

Since $I$ is admissible, $R:=G \cup\left(\mathbb{N} \backslash\left\{n_{k}: k \geq k_{0}\right\}\right)$ is again an element of $I$ and we have

$$
\left\{n \in \mathbb{N}: D_{n i}(P) \geq \varepsilon\right\} \subseteq R \quad \forall i \in S .
$$

Thus we have shown that $D_{n i}(P)$ converges along $I$ to zero uniformly in $i \in S$, which means exactly that $P \in J_{\mathscr{B}, I}$.

In the second case, $\inf _{i \in S} D_{n_{k_{0}} i}(P) \geq 2 / 3$, one can show analogously that $Q \in J_{\mathscr{B}, I}$.

Note that if $I=I_{f}$ and $\inf _{i \in S} \sum_{l=1}^{\infty}\left|b_{n l}^{(i)}-b_{n+1 l}^{(i)}\right|<1 / 3$ for all but finitely many $n \in \mathbb{N}$, then we can take $\mathscr{W}=\{\{n \in \mathbb{N}: n \geq$ $m\}: m \in \mathbb{N}\}$ and condition (91) is satisfied. For the Cesàro matrix $C$ we even have $\lim _{n \rightarrow \infty} \sum_{l=1}^{\infty}\left|c_{n l}-c_{n+1 l}\right|=0$.

As in [24], we can now use the above lemma to obtain a sufficient condition for $\mathscr{B}^{I}$-statistical convergence.

Theorem 26. Under the same general hypotheses as in the previous lemma, if $s=\left(s_{n}\right)_{n \in \mathbb{N}}$ is a $J_{\mathscr{B}, I}$-bounded $\mathscr{B}_{+}^{I}$-statistically pre-Cauchy sequence in $\mathbb{R}$ such that the set $Z$ of all $J_{\mathscr{B}, I^{-}}$ cluster points of $s$ is nowhere dense (note that $Z$ is closed ( $c f$. [1, Theorem 4.1(i)]), so " $Z$ nowhere dense" just means that $Z$ has empty interior) in $\mathbb{R}$, then $s$ is $\mathscr{B}^{I}$-statistically convergent.

Proof. Suppose that $s$ is $J_{\mathscr{B}, I}$-bounded and $\mathscr{B}_{+}^{I}$-statistically pre-Cauchy but not $\mathscr{B}^{I}$-statistically convergent.

As mentioned before, the $J_{\mathscr{B}, I}$-boundedness assures that there is some $a \in Z$. Since $s$ is not $\mathscr{B}^{I}$-statistically convergent there is an $\varepsilon>0$ such that $\left\{n \in \mathbb{N}: s_{n} \leq a-\varepsilon\right\} \notin J_{\mathscr{B}, I}$ or $\left\{n \in \mathbb{N}: s_{n} \geq a+\varepsilon\right\} \notin J_{\mathscr{B}, I}$. Without loss of generality, we assume the former.

As in [24], we will show that $(a-\varepsilon, a) \subseteq Z$. If not, there would be an open interval $(\alpha, \beta) \subseteq(a-\varepsilon, a)$ such that $\{n \in$ $\left.\mathbb{N}: s_{n} \in(\alpha, \beta)\right\} \in J_{\mathscr{B}, I}$.

It follows from Lemma 25 that $X=\left\{n \in \mathbb{N}: s_{n} \leq \alpha\right\} \in$ $J_{\mathscr{B}, I}$ or $Y:=\left\{n \in \mathbb{N}: s_{n} \geq \beta\right\} \in J_{\mathscr{B}, I}$.

Since $X \supseteq\left\{n \in \mathbb{N}: s_{n} \leq a-\varepsilon\right\} \notin J_{\mathscr{B}, I}$, we would have $Y \in J_{\mathscr{B}, I}$. But we can find $\delta>0$ with $\beta<a-\delta$ and because of $a \in Z$ the set $\left\{n \in \mathbb{N}: s_{n}>a-\delta\right\}$ cannot belong to $J_{\mathscr{B}, I}$ where on the other hand it is contained in $Y$.

Thus $Z$ has nonempty interior and the proof is finished.

As an immediate consequence of Theorem 26 we get the following corollary.

Corollary 27. Under the same general assumptions as in Lemma 25 , if s is a $\mathscr{B}_{+}^{I}$-statistically pre-Cauchy sequence in $\mathbb{R}$ whose range is finite, then $s$ is $\mathscr{B}^{I}$-statistically convergent.

\section{A Sup-Limsup-Theorem}

In this section we will present the generalisation of Simons' equality that was announced in the abstract, but first we need to recall some definitions: a boundary for a real Banach space $X$ is a subset $H$ of $B_{X^{*}}$ (for every Banach space $Y$ we denote by $B_{Y}$ its closed unit ball and by $S_{Y}$ its unit sphere) such that for every $x \in X$ there is some $x^{*} \in H$ with $x^{*}(x)=\|x\|$. By the Hahn-Banach-theorem, $S_{X^{*}}$ is always a boundary for $X$. It easily follows from the Krein-Milman-theorem that ex $B_{X^{*}}$, the set of extreme points of $B_{X^{*}}$, is also a boundary for $X$.

A famous theorem due to Rainwater (cf. [25]) states that a bounded sequence in $X$ which is convergent to some $x \in X$ under every functional from ex $B_{X^{*}}$ is weakly convergent to $x$.

Later Simons (cf. $[26,27])$ generalised this result to an arbitrary boundary $H$ by proving that for every bounded sequence $\left(x_{n}\right)_{n \in \mathbb{N}}$ in $X$ the equality

$$
\sup _{x^{*} \in H} \lim \sup x^{*}\left(x_{n}\right)=\sup _{x^{*} \in B_{X^{*}}} \lim \sup x^{*}\left(x_{n}\right)
$$

which is nowadays known as Simons' equality, holds.

An easy separation argument shows that every boundary $H$ satisfies $B_{X^{*}}=\overline{\mathrm{co}} w^{*} H$, but $B_{X^{*}}=\overline{\mathrm{co}} H$ is not true in general (here co $A$ denotes the convex hull, $\bar{A}^{w^{*}}$ the weak ${ }^{*}$-closure, and $\bar{A}$ the norm-closure of $A \subseteq X^{*}$ ).

In [28] Fonf and Lindenstrauss introduced the following intermediate notion. Consider a convex weak ${ }^{*}$-compact subset $K$ of $X^{*}$ (where $X$ is a real or complex Banach space). A subset $H$ of $K$ is said to $(I)$-generate $K$ provided that whenever $H$ is written as a countable union $H=\bigcup_{m=1}^{\infty} H_{m}$, then

$$
\overline{\mathrm{co}}\left(\bigcup_{m=1}^{\infty} \overline{\mathrm{co}}^{w^{*}} H_{m}\right)=K
$$


or equivalently, whenever $H$ is written as a countable union $H=\bigcup_{m=1}^{\infty} H_{m}$ with $H_{m} \subseteq H_{m+1}$, then

$$
\overline{\bigcup_{m=1}^{\infty} \overline{\mathrm{co}^{*}} H_{m}}=K .
$$

Clearly, $K=\overline{\mathrm{co}} H$ implies that $H(I)$-generates $K$ which in turn implies $K=\overline{\mathrm{co}}^{w^{*}} H$, but the converses are not true in general as was shown in [28]. It was also proved in [28] that, for a real Banach space, every boundary of $K(I)$-generates $K$ (the set $H$ is called a boundary of $K$ if $\max \left\{x^{*}(x): x^{*} \in\right.$ $H\}=\sup \left\{x^{*}(x): x^{*} \in K\right\}$ for every $x \in X$. In this terminology, $H$ is a boundary for $X$ if and only if it is a boundary of $\left.B_{X^{*}}\right)$.

Nygaard proved in [29] that Rainwater's theorem holds true for every $(I)$-generating subset of $B_{X^{*}}$ and the authors of [30] showed that Simons' equality is equivalent to the (I)-generation property (cf. [30, Theorem 2.2]; see also [31, Lemma 2.1 and Remark 2.2]).

In [32] the author investigated the possibility to generalise the Rainwater-Simons-convergence theorem for $(I)$ generating sets to some generalised convergence methods such as strong $A$-p-summability and almost convergence by proving a general Simons-like inequality for $(I)$-generating sets (cf. [32, Theorem 3.1]). We will continue this work here, using similar arguments as in [32] to generalise Simons' equality to the $J_{\mathscr{B}, I}$-limsup for the case that $\mathscr{F}(I)$ has a countable base, and obtain some related convergence results.

First we need the following lemma, whose proof is-once more-analogous to those of Propositions 3 and 4. Therefore, the details will be skipped.

Lemma 28. Let each $B_{i}$ be nonnegative. Define $f: \mathbb{R} \rightarrow$ $[0, \infty)$ by $f(t)=t$ for $t \geq 0$ and $f(t)=0$ for $t<0$. Put $A(s, a, \varepsilon):=\left\{k \in \mathbb{N}: s_{k}>a+\varepsilon\right\}$ for every $\varepsilon>0$. Then

$$
\begin{gathered}
I-\lim \sum_{k=1}^{\infty} b_{n k}^{(i)} f\left(s_{k}-a\right)=0 \quad \text { uniformlyini } \in S \\
\Longrightarrow A(s, a, \varepsilon) \in J_{\mathscr{R}, I} \quad \forall \varepsilon>0
\end{gathered}
$$

and the converse is true if the sequences is bounded and

$$
\sup \left\{\sum_{k=1}^{\infty} b_{n k}^{(i)}: n \in \mathbb{N} \backslash A, i \in S\right\}<\infty
$$

for some $A \in I$.

Now we turn to the generalisation of Simons' equality.

Theorem 29. Let $X$ be a real Banach space, $K \subseteq X^{*}$ a convex weak ${ }^{*}$-compact subset, and $H \subseteq K$ an (I)-generating set for $K$. Let the ideal $I$ be such that the filter $\mathscr{F}(I)$ has a countable base. Assume that each $B_{i}$ is nonnegative and that there exists an $A \in I$ such that

$$
M:=\sup \left\{\sum_{k=1}^{\infty} b_{n k}^{(i)}: n \in \mathbb{N} \backslash A, i \in S\right\}<\infty .
$$

Let $\left(x_{n}\right)_{n \in \mathbb{N}}$ be a bounded sequence in $X$. Then the equality

$$
\sup _{x^{*} \in H} J_{\mathscr{B}, I}-\lim \sup x^{*}\left(x_{n}\right)=\sup _{x^{*} \in K} J_{\mathscr{B}, I}-\lim \sup x^{*}\left(x_{n}\right)
$$

holds.

Proof. Denote the left-hand supremum by $c$ and the righthand supremum by $d$. We only have to show $d \leq c$. Let $R=\sup _{n \in \mathbb{N}}\left\|x_{n}\right\|$. Let $\left(C_{n}\right)_{n \in \mathbb{N}}$ be a countable base for $\mathscr{F}(I)$. Without loss of generality we may assume $C_{n+1} \subseteq C_{n}$ for all $n$. Take $x^{*} \in K$ and $\varepsilon>0$ arbitrary and put

$$
\begin{gathered}
E_{m}=\left\{y^{*} \in K: \sum_{k=1}^{\infty} b_{n k}^{(i)} f\left(y^{*}\left(x_{k}\right)-c\right)\right. \\
\left.\leq \varepsilon \forall i \in S, n \in C_{m}\right\}, \\
H_{m}=E_{m} \cap H \quad \forall m \in \mathbb{N},
\end{gathered}
$$

where $f$ is as in the previous lemma. Then $H_{m} \subseteq H_{m+1}$ for every $m \in \mathbb{N}$. It follows from [21, Theorem 1] that $\{n \in \mathbb{N}$ : $\left.y^{*}\left(x_{n}\right)>c+\delta\right\} \in J_{\mathscr{B}, I}$ for every $\delta>0$. Together with the previous lemma this easily implies $\bigcup_{m=1}^{\infty} H_{m}=H$.

Since $H(I)$-generates $K$, we get that

$$
K=\overline{\bigcup_{m=1}^{\infty} \overline{\operatorname{co}} w^{*} H_{m}}
$$

Thus we can find $m \in \mathbb{N}$ and $y^{*} \in \overline{\mathrm{co}}^{w^{*}} H_{m}$ with $\left\|x^{*}-y^{*}\right\| \leq \varepsilon$. It is easily checked that $E_{m}$ is convex and weak ${ }^{*}$-closed; hence $y^{*} \in E_{m}$. But for every $k \in \mathbb{N}$

$$
\begin{aligned}
f\left(x^{*}\left(x_{k}\right)-c\right) \leq & f\left(x^{*}\left(x_{k}\right)-y^{*}\left(x_{k}\right)\right) \\
& +f\left(y^{*}\left(x_{k}\right)-c\right) \\
\leq & \left\|x^{*}-y^{*}\right\|\left\|x_{k}\right\|+f\left(y^{*}\left(x_{k}\right)-c\right) \\
\leq & R \varepsilon+f\left(y^{*}\left(x_{k}\right)-c\right) .
\end{aligned}
$$

It follows that

$$
\begin{aligned}
\sum_{k=1}^{\infty} b_{n k}^{(i)} f\left(x^{*}\left(x_{k}\right)-c\right) & \leq M R \varepsilon+\sum_{k=1}^{\infty} b_{n k}^{(i)} f\left(y^{*}\left(x_{k}\right)-c\right) \\
& \leq \varepsilon(M R+1)
\end{aligned}
$$

for every $i \in S$ and every $n \in C_{m} \cap(\mathbb{N} \backslash A)$. Since $C_{m} \cap(\mathbb{N} \backslash A) \epsilon$ $\mathscr{F}(I)$ and $\varepsilon>0$ was arbitrary, we conclude with Lemma 28 that $\left\{n \in \mathbb{N}: x^{*}\left(x_{n}\right)>c+\delta\right\} \in J_{\mathscr{B}, I}$ for every $\delta>0$, whence $J_{\mathscr{B}, I}-\lim \sup x^{*}\left(x_{n}\right) \leq c$.

As a corollary, we get the following convergence result.

Corollary 30. Under the same hypotheses as in Theorem 29 with $K=B_{X^{*}}$, if $x \in X$ is such that $\left(x^{*}\left(x_{n}\right)\right)_{n \in \mathbb{N}}$ is $\mathscr{B}^{I}$ statistically convergent to $x^{*}(x)$ for every $x^{*} \in H$ then the same holds true for every $x^{*} \in X^{*}$; that is, $\left(x_{n}\right)_{n \in \mathbb{N}}$ is "weakly $\mathscr{B}^{I}$ statistically convergent to $x . "$ 
Moreover, for every family $\mathscr{F}=\left(F_{k}^{(i)}\right)_{k \in \mathbb{N}, i \in S}$ in $\mathscr{M} \cup \mathcal{O}$ which is equicontinuous at 0 and satisfies

$$
\begin{gathered}
\inf \left\{F_{k}^{(i)}(t): k \in \mathbb{N}, i \in S\right\}>0 \quad \forall t>0, \\
\sup \left\{F_{k}^{(i)}(t): k \in \mathbb{N}, i \in S\right\}<\infty \quad \forall t \geq 0
\end{gathered}
$$

$\left(x^{*}\left(x_{n}\right)\right)_{n \in \mathbb{N}}$ is strongly $\mathscr{B}^{I}$-summable to $x^{*}(x)$ with respect to $\mathscr{F}$ for every $x^{*} \in X^{*}$ whenever this statement holds for every $x^{*} \in H$.

Proof. The first statement follows directly from Theorem 29 and the second follows from the first one via Propositions 3 and 4 .

It is clear that this convergence result carries over to complex Banach spaces (note that if $X$ is a complex Banach space and $H(I)$-generates $B_{X^{*}}$; then $\left\{\operatorname{Re} x^{*}: x^{*} \in H\right\}(I)$ generates $\left\{\operatorname{Re} x^{*}: x^{*} \in B_{X^{*}}\right\}$, the unit ball of the underlying real space).

In particular, if we take each $B_{i}$ to be the infinite unit matrix, we get that, for every ideal $I$ such that $\mathscr{F}(I)$ has a countable base, $I$ - $\lim x^{*}\left(x_{n}\right)=x^{*}(x)$ for every $x^{*} \in X^{*}$ whenever this is true for every $x^{*}$ in an $(I)$-generating subset of $B_{X^{*}}$ (in particular, in a boundary for $X$ ). We can also prove an analogous convergence result for $\mathscr{B}^{I}$-summability.

Proposition 31. Let $X$ be a real or complex Banach space and $H \subseteq B_{X^{*}}$ an (I)-generating set for $B_{X^{*}}$. Suppose that $\mathscr{F}(I)$ has a countable base, $\sum_{k=1}^{\infty}\left|b_{n k}^{(i)}\right|<\infty$ for all $n \in \mathbb{N}, i \in S$, and moreover

$$
M:=\sup \left\{\sum_{k=1}^{\infty}\left|b_{n k}^{(i)}\right|: n \in \mathbb{N} \backslash A, i \in S\right\}<\infty
$$

for some $A \in I$.

Let $\left(x_{n}\right)_{n \in \mathbb{N}}$ be a bounded sequence in $X$ and $x \in X$ such that $\left(x^{*}\left(x_{n}\right)\right)_{n \in \mathbb{N}}$ is $\mathscr{B}^{I}$-summable to $x^{*}(x)$ for every $x^{*} \in H$. Then the same is true for every $x^{*} \in X^{*}$.

Proof. Let $\left(C_{n}\right)_{n \in \mathbb{N}}$ be a decreasing countable basis for $\mathscr{F}(I)$. Let $R \geq \sup _{n \in \mathbb{N}}\left\|x_{n}\right\|$ and $R \geq\|x\|$. Take any $x^{*} \in B_{X^{*}}$ and fix an arbitrary $\varepsilon>0$. Define

$$
\begin{gathered}
E_{m}:=\left\{y^{*} \in B_{X^{*}}: \sup _{i \in S}\left|\sum_{k=1}^{\infty} b_{n k}^{(i)} y^{*}\left(x_{k}\right)-y^{*}(x)\right|\right. \\
\left.\leq \varepsilon \forall n \in C_{m}\right\}, \\
H_{m}:=E_{m} \cap H \quad \forall m \in \mathbb{N} .
\end{gathered}
$$

Then $H_{m} \nearrow H$ and since $H(I)$-generates $B_{X^{*}}$, we can find $m \in \mathbb{N}$ and $y^{*} \in \overline{\mathrm{co}^{*}} H_{m}$ such that $\left\|x^{*}-y^{*}\right\| \leq \varepsilon$.
It is not too hard to see that $E_{m}$ is convex and weak ${ }^{*}$ closed and thus $y^{*} \in E_{m}$. Consequently, for all $i \in S$ and $n \in C_{m} \cap(\mathbb{N} \backslash A)$ we have

$$
\begin{aligned}
\left|\sum_{k=1}^{\infty} b_{n k}^{(i)} x^{*}\left(x_{k}\right)-x^{*}(x)\right| \leq & \left|\sum_{k=1}^{\infty} b_{n k}^{(i)}\left(x^{*}\left(x_{k}\right)-y^{*}\left(x_{k}\right)\right)\right| \\
& +\left|\sum_{k=1}^{\infty} b_{n k}^{(i)} y^{*}\left(x_{k}\right)-y^{*}(x)\right| \\
& +\left|y^{*}(x)-x^{*}(x)\right| \\
\leq & M\left\|x^{*}-y^{*}\right\| R+\varepsilon+\left\|x^{*}-y^{*}\right\| R \\
\leq & \varepsilon(R(M+1)+1) .
\end{aligned}
$$

Since $C_{m} \cap(\mathbb{N} \backslash A) \in \mathscr{F}(I)$ and $\varepsilon>0$ was arbitrary, we are done.

The next result concerning $\mathscr{B}^{I}$-statistically pre-Cauchy sequences is a generalisation of [32, Corollary 3.5]. Using Propositions 21 and 22 with $F_{k l}^{(i)}=\operatorname{id}_{[0, \infty)}$ for all $k, l \in \mathbb{N}$ and $i \in S$, its proof can be carried out analogously to that of Proposition 31. The details will be omitted.

Proposition 32. Let $X$ be a real or complex Banach space and $H \subseteq B_{X^{*}}$ an $(I)$-generating set for $B_{X^{*}}$. Suppose that $\mathscr{F}(I)$ has a countable base, that each $B_{i}$ is nonnegative, and that there is some $A \in I$ such that

$$
\sup \left\{\sum_{k=1}^{\infty} b_{n k}^{(i)}: n \in \mathbb{N} \backslash A, i \in S\right\}<\infty .
$$

Let $\left(x_{n}\right)_{n \in \mathbb{N}}$ be a bounded sequence in $X$ such that $\left(x^{*}\left(x_{n}\right)\right)_{n \in \mathbb{N}}$ is $\mathscr{B}^{I}$-statistically pre-Cauchy and $\mathscr{B}_{+}^{I}$-statistically preCauchy, respectively, for every $x^{*} \in H$. Then the same is true for every $x^{*} \in X^{*}$.

Finally, let us give characterisations of weak-compactness and reflexivity that generalise [32, Corollaries 3.7 and 3.8].

Corollary 33. Let $M$ be a bounded subset of the Banach space $X$ and $B$ an (I)-generating set for $B_{X^{*}}$. Then $M$ is weakly relatively compact if (and only if) for every sequence $\left(x_{n}\right)_{n \in \mathbb{N}}$ in $M$ there is an element $x \in X$, an ideal I on $\mathbb{N}$ such that $\mathscr{F}(I)$ admits a countable base, and a nonnegative matrix $A=$ $\left(a_{n k}\right)_{n, k \geq 1}$ such that

$$
\begin{gathered}
\exists C \in I \sup _{n \in \mathbb{N} \backslash C} \sum_{k=1}^{\infty} a_{n k}<\infty, \\
I-\lim a_{n k}=0 \quad \forall k \in \mathbb{N}
\end{gathered}
$$

and $\left(x^{*}\left(x_{n}\right)\right)_{n \in \mathbb{N}}$ is $A^{I}$-statistically convergent to $x^{*}(x)$ for every $x^{*} \in B$.

Proof. Let $\left(x_{n}\right)_{n \in \mathbb{N}}$ be an arbitrary sequence in $M$ and fix $x, I$, and $A$ as above. By Corollary $30\left(x^{*}\left(x_{n}\right)\right)_{n \in \mathbb{N}}$ is $A^{I}$ statistically convergent to $x^{*}(x)$ for every $x^{*} \in X^{*}$. Thus, 
given finitely many functionals $x_{1}^{*}, \ldots, x_{m}^{*} \in X^{*}$, the sequence $\left(\sum_{j=1}^{m}\left|x_{j}^{*}\left(x_{n}-x\right)\right|\right)_{n \in \mathbb{N}}$ is $A^{I}$-statistically convergent to zero. Hence for any $\varepsilon>0$ the set $D_{\varepsilon}=\left\{n \in \mathbb{N}: \sum_{j=1}^{m} \mid x_{j}^{*}\left(x_{n}-\right.\right.$ $x) \mid<\varepsilon\}$ does not belong to $J_{A, I}$.

By (119), $J_{A, I}$ is admissible; therefore, $D_{\varepsilon}$ must be infinite for every $\varepsilon>0$, which shows that $x$ is a weak-cluster point of $\left(x_{n}\right)_{n \in \mathbb{N}}$.

So $M$ is weakly relatively and countably compact and by the Eberlein-Shmulyan theorem, it must be also weakly relatively compact.

Corollary 34. If $B_{X}$ is an (I)-generating set for $B_{X^{* *}}$ (we consider $X$ canonically embedded into its bidual), then $X$ is reflexive if (and only if) for every sequence $\left(x_{n}^{*}\right)_{n \in \mathbb{N}}$ in $B_{X^{*}}$ there is a functional $x^{*} \in X^{*}$, an ideal I on $\mathbb{N}$ such that $\mathscr{F}(I)$ admits a countable base, and a nonnegative matrix A such that (118) and (119) are satisfied and $\left(x_{n}^{*}(x)\right)_{n \in \mathbb{N}}$ is $A^{I}$-statistically convergent to $x^{*}(x)$ for every $x \in X$.

Proof. By the previous corollary, $B_{X^{*}}$ is weakly compact; thus $X^{*}$ and hence also $X$ are reflexive.

\section{Conflict of Interests}

The author declares that there is no conflict of interests regarding the publication of this paper.

\section{Acknowledgment}

The author is grateful to the anonymous referee for pointing out reference [16] and the final version of [19].

\section{References}

[1] P. Kostyrko, T. Šalát, and W. Wilczyński, "I-convergence," Real Analysis Exchange, vol. 26, no. 2, pp. 669-685, 2000/01.

[2] B. K. Lahiri and P. Das, " $I$ and $I^{x} 2 a$;-convergence in topological spaces," Mathematica Bohemica, vol. 130, no. 2, pp. 153-160, 2005.

[3] P. Kostyrko, M. Mačaj, T. Šalát, and M. Sleziak, "I-convergence and extremal I-limit points," Mathematica Slovaca, vol. 55, no. 4, pp. 443-464, 2005.

[4] K. Dems, "On I-Cauchy sequences," Real Analysis Exchange, vol. 30, no. 1, pp. 123-128, 2004.

[5] H. Fast, "Sur la convergence statistique," Colloquium Mathematicae, vol. 2, no. 3-4, pp. 241-244, 1951 (French).

[6] J. S. Connor, "The statistical and strong $p$-Cesàro convergence of sequences," Analysis, vol. 8, no. 1-2, pp. 47-63, 1988.

[7] J. Connor, "Two valued measures and summability," Analysis, vol. 10, no. 4, pp. 373-385, 1990.

[8] K. Zeller and W. Beekmann, Theorie der Limitierungsverfahren, Springer, Berlin, Germany, 2nd edition, 1970, (German).

[9] H. J. Hamilton and J. D. Hill, "On strong summability," American Journal of Mathematics, vol. 60, no. 3, pp. 588-594, 1938.

[10] I. J. Maddox, "Spaces of strongly summable sequences," The Quarterly Journal of Mathematics, vol. 18, no. 2, pp. 345-355, 1967.
[11] K. Demirci, "Strong $A$-summability and $A$-statistical convergence," Indian Journal of Pure and Applied Mathematics, vol. 27, no. 6, pp. 589-593, 1996.

[12] G. G. Lorentz, "A contribution to the theory of divergent sequences," Acta Mathematica, vol. 80, no. 1, pp. 167-190, 1948.

[13] M. Stieglitz, "Eine verallgemeinerung des begriffs der fastkonvergenz," Mathematica Japonica, vol. 18, pp. 53-70, 1973 (German).

[14] I. J. Maddox, “On strong almost convergence," Mathematical Proceedings of the Cambridge Philosophical Society, vol. 85, no. 2, pp. 345-350, 1979.

[15] E. Savas, P. Das, and S. Dutta, "A note on strong matrix summability via ideals," Applied Mathematics Letters, vol. 25, no. 4, pp. 733-738, 2012.

[16] O. Duman and C. Orhan, " $\mu$-statistically convergent function sequences," Czechoslovak Mathematical Journal, vol. 54, no. 2 , pp. 413-422, 2004.

[17] J. Connor, "On strong matrix summability with respect to a modulus and statistical convergence," Canadian Mathematical Bulletin, vol. 32, no. 2, pp. 194-198, 1989.

[18] K. Demirci, “On A-statistical cluster points," Glasnik Matematički III, vol. 37, no. 2, pp. 293-301, 2002.

[19] M. Burgin and O. Duman, "Statistical fuzzy convergence," International Journal of Uncertainty, Fuzziness and KnowledgeBased Systems, vol. 16, no. 6, pp. 879-902, 2008.

[20] J. A. Fridy, “On statistical convergence," Analysis, vol. 5, no. 4, pp. 301-313, 1985.

[21] K. Demirci, "I-limit superior and limit inferior," Mathematical Communications, vol. 6, no. 2, pp. 165-172, 2001.

[22] J. A. Fridy and C. Orhan, "Statistical limit superior and limit inferior," Proceedings of the American Mathematical Society, vol. 125, no. 12, pp. 3625-3631, 1997.

[23] J. A. Fridy, "Statistical limit points," Proceedings of the American Mathematical Society, vol. 118, no. 4, pp. 1187-1192, 1993.

[24] J. Connor, J. Fridy, and J. Kline, "Statistically pre-Cauchy sequences," Analysis, vol. 14, no. 4, pp. 311-317, 1994.

[25] J. Rainwater, "Weak convergence of bounded sequences," Proceedings of the American Mathematical Society, vol. 14, p. 999, 1963.

[26] S. Simons, "A convergence theorem with boundary," Pacific Journal of Mathematics, vol. 40, pp. 703-708, 1972.

[27] S. Simons, "An eigenvector proof of Fatou's lemma for continuous functions," The Mathematical Intelligencer, vol. 17, no. 3, pp. 67-70, 1995.

[28] V. P. Fonf and J. Lindenstrauss, "Boundaries and generation of convex sets," Israel Journal of Mathematics, vol. 136, pp. 157-172, 2003.

[29] O. Nygaard, "A remark on Rainwater's theorem," Annales Mathematicae et Informaticae, vol. 32, pp. 125-127, 2005.

[30] B. Cascales, V. P. Fonf, J. Orihuela, and S. Troyanski, "Boundaries of Asplund spaces," Journal of Functional Analysis, vol. 259, no. 6, pp. 1346-1368, 2010.

[31] O. F. K. Kalenda, “(I)-envelopes of closed convex sets in Banach spaces," Israel Journal of Mathematics, vol. 162, no. 1, pp. 157-181, 2007.

[32] J.-D. Hardtke, "Rainwater-Simons type convergence theorems for generalized convergence methods," Acta et Commentationes Universitatis Tartuensis de Mathematica, no. 14, pp. 65-74, 2010. 


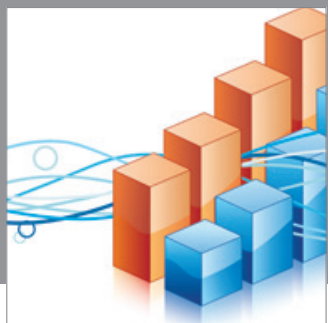

Advances in

Operations Research

mansans

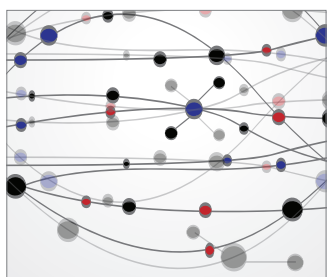

The Scientific World Journal
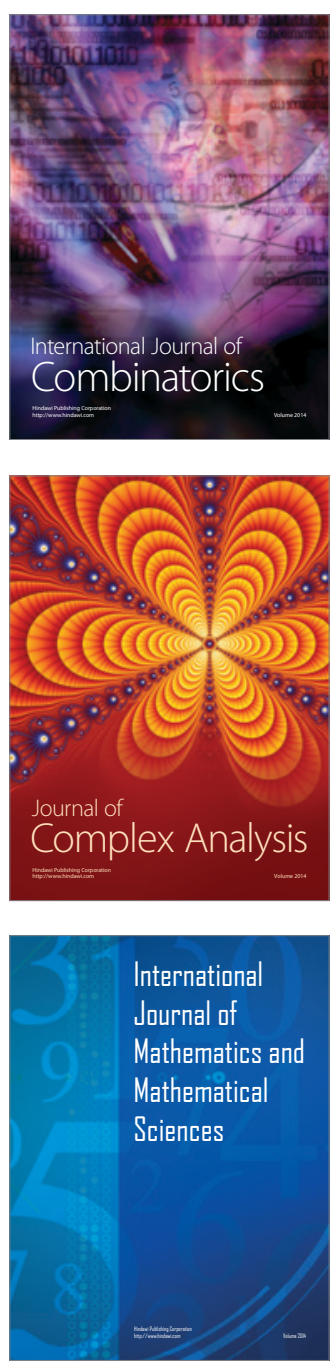
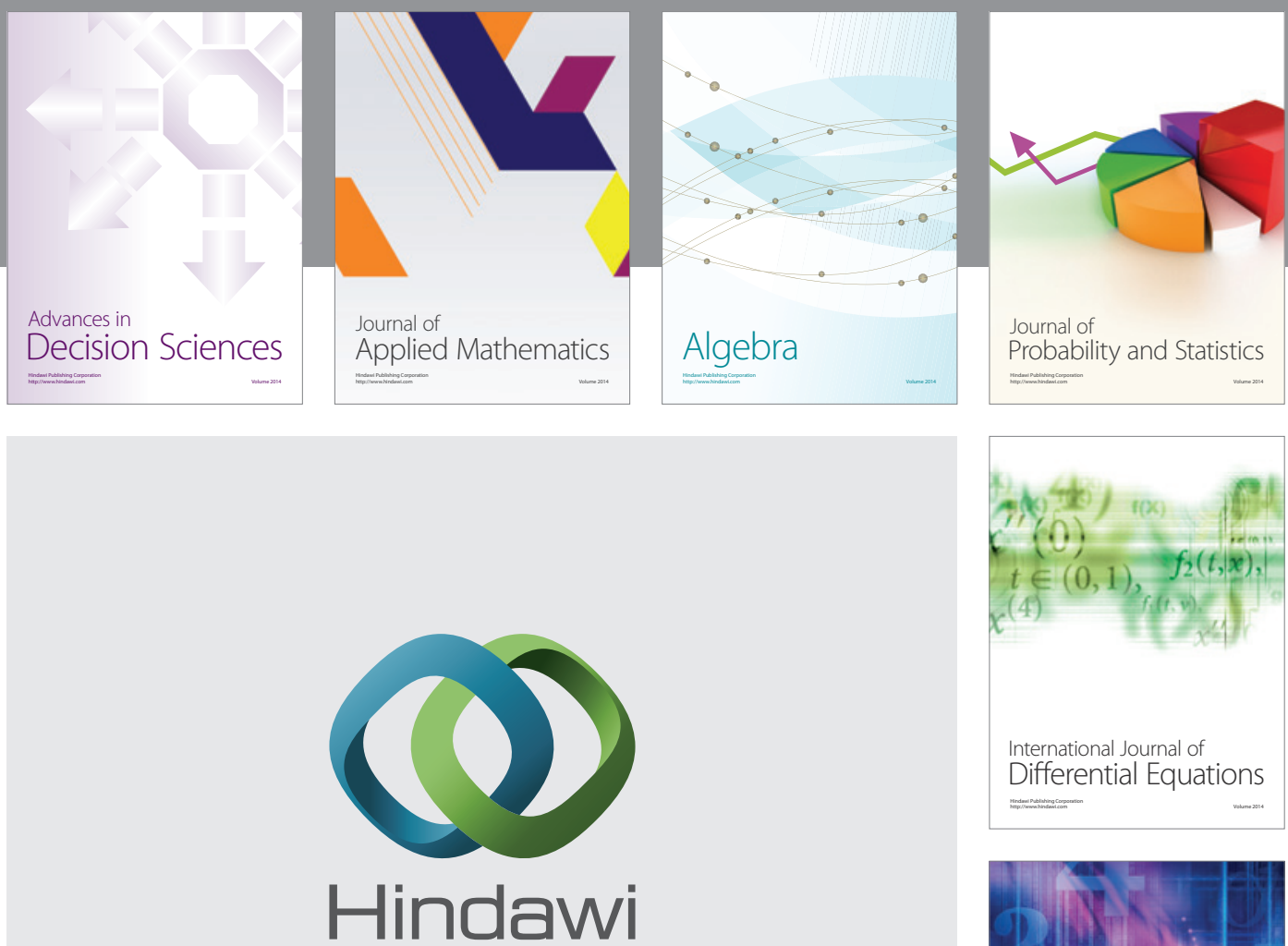

Submit your manuscripts at http://www.hindawi.com
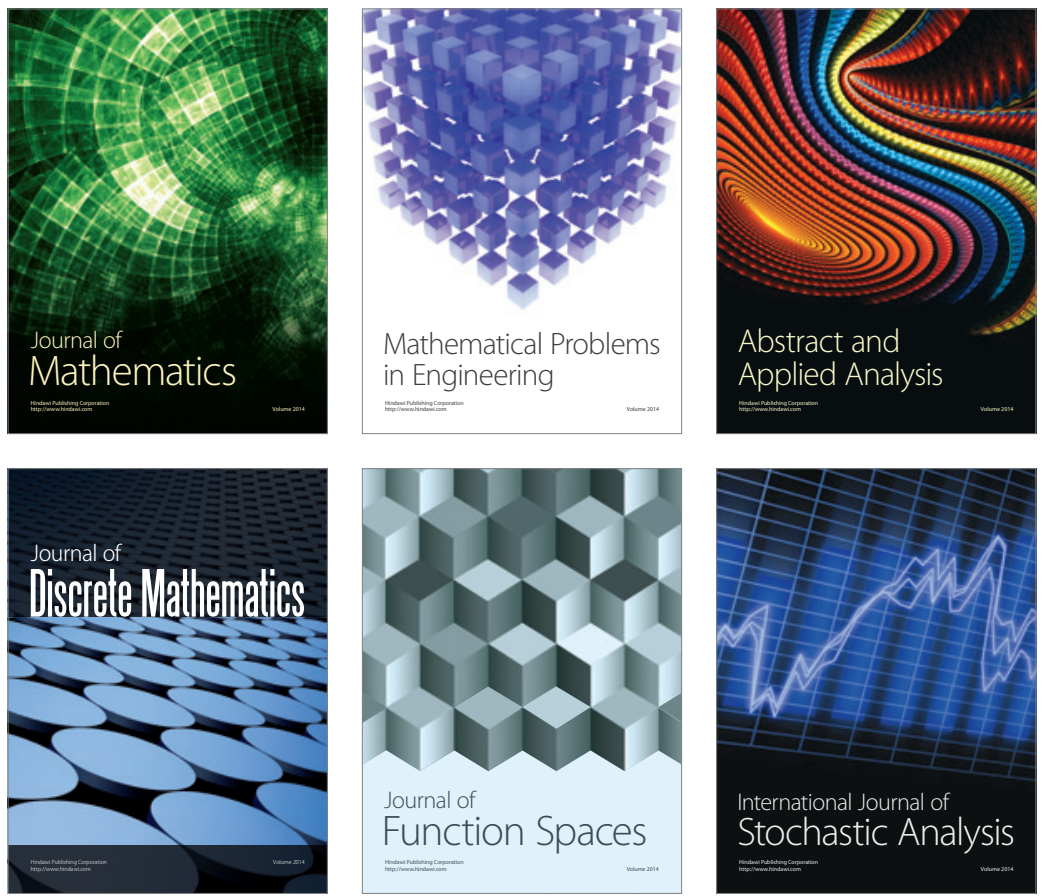

Journal of

Function Spaces

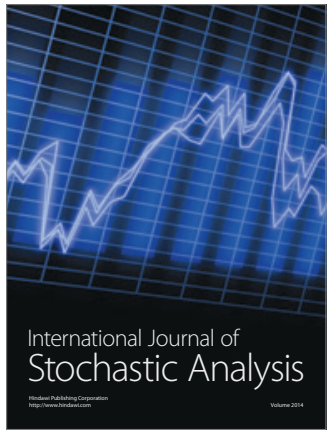

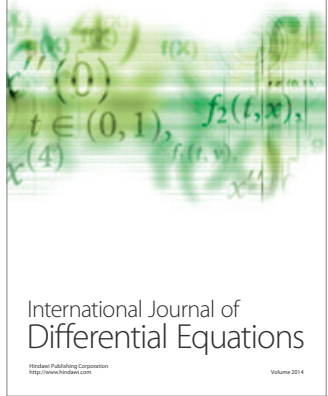
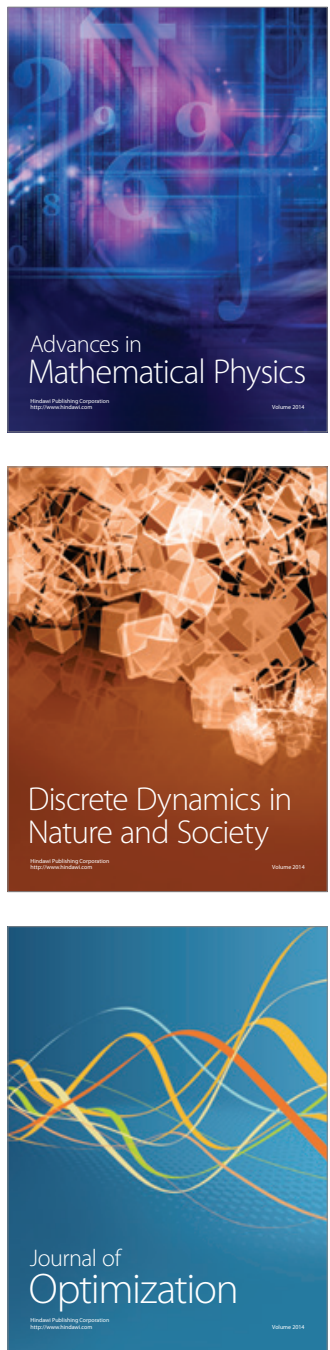